\title{
Nonlinear Attitude Control of a Spacecraft with Distributed Actuation of Solar Arrays
}

\author{
Yashwanth Kumar Nakka*, Soon-Jo Chung ${ }^{\dagger}$ \\ California Institute of Technology, Pasadena, CA, 91125, USA \\ James T. Allison ${ }^{\ddagger}$ \\ University of Illinois at Urbana-Champaign, Urbana, IL, 61801, USA \\ Jack B. Aldrich\$, and Oscar S. Alvarez-Salazar $₫$ \\ Jet Propulsion Laboratory, California Institute of Technology, Pasadena, CA, 91109, USA
}

This article presents a novel control architecture and algorithm for precision attitude control of a one-degree-of-freedom dynamic model of a spacecraft. To achieve a parametric model-based control design approach for this new spacecraft actuation and control architecture, the nonlinear dynamics of the open-loop plant are modeled as an Ordinary Differential Equation (ODE)-Partial Differential Equation (PDE) system. The ODE describes the spacecraft single-axis rigid-body rotation, and the PDE describes the spatially continuous flexible dynamics of the solar array including an allocation for a multi-input distributed piezoelectric actuation system bonded on the solar array. This distributed actuation system is called strain-actuated solar arrays. Based on this plant model, a nonlinear ODE-PDE feedback controller for attitude trajectory tracking and slewing is presented with detailed stability proofs. From an end-to-end point of view, the controller drives the distributed piezoelectric actuator patches with voltages that induce bending deflections in the solar arrays, causing controlled reaction torques on the bus to yield target-following motions and precision spacecraft attitude control. The proposed algorithm can be extended to any distributed actuation system with appropriate control input to actuator input mapping. The benefits and limitations of the proposed attitude control method using strain actuation

\footnotetext{
*Graduate Student, Graduate Aerospace Laboratories (GALCIT), ynakka@caltech.edu

$\dagger$ Associate Professor and Bren Scholar, Graduate Aerospace Laboratories (GALCIT), sjchung@caltech.edu. AIAA Senior Member

$¥$ Assistant Professor, Department of Industrial and Enterprise Systems Engineering, jtalliso@illinois.edu. AIAA Member

§Senior Member, Guidance and Control Analysis Group, Jack.B.Aldrich@jpl.nasa.gov. AIAA Member.

๑Manager, Guidance and Control Analysis Group, Oscar.S.Alvarez-Salazar@jpl.nasa.gov. AIAA Member.
} 
are discussed later in terms of solar array inertia and structural rigidity. This paper also reports experimental results that demonstrate command-following rotations of a cylindrical bus via closed-loop control of its flexible appendages.

\section{Nomenclature}

$$
\begin{aligned}
& r \quad=\quad \text { spacecraft bus radius }-m \\
& \ell \quad=\text { length of the solar array }-m \\
& \xi \quad=\text { displacement due to bending in the solar array }-m \\
& \theta \quad=\text { rotation of the spacecraft - radians } \\
& \left(x_{3}, y_{3}\right)=\text { inertial coordinate system at the center of the spacecraft } \\
& \rho_{b} \quad=\text { density of the beam }-\mathrm{kg} / \mathrm{m}^{3} \\
& \rho_{p} \quad=\text { density of PZT }-k g / m^{3} \\
& I_{b} \quad=\text { area moment of inertia of the beam }-m^{4} \\
& I_{p} \quad=\text { area moment of inertia of PZT }-m^{4} \\
& A_{b} \quad=\text { area of cross section of the beam }-m^{2} \\
& A_{p} \quad=\quad \text { area of cross section of PZT }-m^{2} \\
& E_{b} \quad=\text { Young's modulus of the beam }-N / m^{2} \\
& E_{p} \quad=\text { Young's modulus of PZT }-N / m^{2} \\
& t_{b} \quad=\text { thickness of the beam }-m \\
& t_{p} \quad=\text { thickness of PZT }-m \\
& m_{b} \quad=\text { mass per unit length of the beam }-\mathrm{kg} / \mathrm{m} \\
& m_{p} \quad=\quad \text { mass per unit length of } \mathrm{PZT}-\mathrm{kg} / \mathrm{m} \\
& m_{v} \quad=\text { point mass }-k g \\
& h_{n} \quad=\text { distance between the top surface of the composite beam to the neutral axis }-m \\
& V \quad=\quad \text { voltage applied across the PZT }-V \\
& d_{x z}, d_{31}=\text { piezoelectric coefficients } \\
& \epsilon \quad=\quad \text { strain profile across the composite beam } \\
& \epsilon_{p} \quad=\text { strain in an unconstrained PZT when a voltage } V \text { is applied } \\
& J_{\theta} \quad=\text { mass moment of inertia of the spacecraft }-\mathrm{kgm}^{2} \\
& E I \quad=\text { total rigidity of the composite beam }-\mathrm{Nm}^{2}
\end{aligned}
$$




$\begin{array}{ll}W_{\mathrm{nc}} & =\text { nonconservative work done }-J \\ M_{b} & =\text { moment applied at a cross section of the beam }-N m \\ \mathcal{L}_{2} & =\text { spatial norm } \\ \sigma_{1} & =\text { lower bound of the inertia matrix } \\ \sigma_{2} & =\text { upper bound of the inertia matrix } \\ \theta_{r} & =\text { reference attitude signal - radian } \\ \xi_{r} & =\text { deference beam bending }-m \\ \theta_{d} & =\text { gains } \\ K_{\theta}, K_{\xi}, \lambda_{\theta} & =\text { Galerkin functions } \\ \phi & =\text { step function } \\ u & =\text { Dirac delta function } \\ \delta_{d} & \end{array}$

\section{Introduction}

Space observatories require precise attitude control and pointing accuracy to achieve desired diffractionlimited optical performance. For example, the Hubble and James Webb Space Telescopes require 7 milliarcsec [1] and 15 milliarcsec [2] pointing accuracies, respectively. A range of emerging science missions such as Asteria [3], Exo-C 4] and technologies such as laser communication [5], precision formation flying [6], and interferometric imaging [7, place stringent requirements on pointing error and stability. In these state-ofthe-art space observatories, precision imaging involves two stages [4, 8]: (i) coarse stage instrument pointing, which is achieved using the primary spacecraft Attitude Control System (ACS), and (ii) fine stage precision control and jitter reduction. Coarse stages are typically built from Reaction Wheel Assemblies (RWAs), because hydrazine thrusters are too disruptive for precision imaging [2]. In this case, however, the RWA system, which drives the ACS, ironically becomes the dominant source of instrument jitter and imaging performance degradation. To avoid these issues, the RWA disturbance must be eliminated at its source, so that an ultra-quiet, spacecraft actuation system (UQSAS), i.e., one that does not produce severe unwanted disturbances as a side effect, can be put in its place. Recently, electric propulsion systems have been proposed as viable UQSAS options, but these actuation systems are clearly fuel-limited and are typically designed as low-thrust propulsion systems that do not have enough control authority to support a high-bandwidth pointing control loop. In contrast, Strain-Actuated Solar Arrays (SASA) have been recently introduced as a propellant-free, UQSAS alternative [9, 10, that can support a high-bandwidth pointing control loop assuming 
a high force-density piezoelectric-based actuation system.

Given the potential for SASA to serve as a viable UQSAS alternative, several studies have been conducted thus far. In [10], replacing the RWA with SASA to achieve spacecraft slewing, attitude control, and momentum management was investigated. In [9, 11, a design optimization of both the control architecture and structural geometry was reported. In contrast to earlier work, this article presents a complete derivation of the spacecraft-SASA dynamics in which the infinite-dimensional nature of the flexible appendage is modeled using Euler-Bernoulli beam theory [12, 13]. More specifically, the nonlinear dynamics of the open-loop plant is modeled as an ODE-PDE system with the ODE describing spacecraft single-axis rigid-body rotation, and the PDE describing the spatially continuous flexible dynamics of the solar array, which includes an allocation for a multi-input distributed piezoelectric actuation system. This study includes a relevant control-structure interaction stability analysis, simulation, and experimental validation. The salient feature of the proposed approach is that the piezoelectric actuators induce bending deflections that are strategically manipulated by a novel underactuated control law to effectively yield the needed array-to-bus coupling (reaction torques) required by the assumed precision pointing tracking control system realization (that is derived herein).

A literature survey aimed at extracting an appropriate open-loop plant model for model-based control design approach revealed several candidate modeling approaches, including those based directly on classical methods such as the Newton-Euler method, Lagrange's equation, Hamilton's principle, and the principal of virtual work, where the spacecraft and flexible appendages are modeled as a rigid/flexible multi-body system [14-17]. These approaches combine finite and infinite-dimensional dynamics via a coupled ODE-PDE system of nonlinear equations, which can be linearized as in [12, 15, 16, 18, In particular, [17] accounted for rotation-induced centrifugal stiffening in the context of a linearized analysis. In this paper, the linear model for a circular bus with flexible appendages in [15] is extended with axial stiffness in the beam due to rotation and gyroscopic terms using the explicit generalization of Lagrange's equation for infinite dimension systems [15]. As such, the resulting system dynamics becomes coupled, nonlinear, and under-actuated in nature [19, 20] with control inputs derived exclusively from the underlying SASA actuation system. This is noteworthy because earlier studies of SASA, including [21], modeled the solar array with a finite-dimensional rigid-body approximation, in contrast to the infinite dimensional PDE model that is used herein. The importance of improving model fidelity in this manner is underscored by the fact that finite-dimensional controllers are prone to spillover [22, 23] when applied to the actual infinite dimensional system for which they were designed.

Numerous previous studies [15, 24, 25] have focused on the control-structure interaction problem for vibration suppression of flexible appendages. However, this paper is the first of its kind where a beam is driven by distributed actuators such that the underactuated spacecraft bus achieves attitude guidance and control 
objectives. In either case, a canonical system of ODE-PDE equations of motion is generated, for which additional processing takes on either of the following two standard methods: (i) the ODE-PDE system is discretized into an ODE system using either Galerkin [12, 13, 26] or operator-theoretic methods [27, or (ii) The ODE-PDE form is left intact [28] for analysis. As mentioned previously, discretization in the first method leads to spillover issues [22, 23]; while keeping PDEs intact avoids this problem.

It is worth noting that ODE-PDE cascaded systems, similar to the SASA system generated herein, have been studied previously in the literature. Published results in this area depend heavily on the type of PDE classification. Indeed, separate results have been obtained for first-order wave equation [29, 30], second-order wave equation [31, and parabolic equation [32, 33. In each of these cases, the control input entering the system at the boundary can assume either a Dirichlet [31, 33] or a Neumann interconnection [34]. In these works, however, the ODE-PDE interaction is strictly unidirectional with regards to how the PDE couples with the ODE system. In contrast, [32] presents an ODE-PDE system where this assumption is relaxed so that the more complete modeling case of bidirectional coupling can be considered. With regards to control, a PDE backstepping method [28, 31, 33] and boundary controller are able to provide infinite-dimensional full-state feedback where the gain kernels are used to compensate for PDE dynamics while stabilization of the entire system is achieved. Similarly, the ODE-PDE SASA system considered herein is bidirectionally coupled with a distributed control input. The open-loop model's inherent passivity [35], and kinetic symmetry [36] are exploited in the construction of the nonlinear ODE-PDE attitude control law. Specifically, the nonlinear controller derivation proposed in [37, 38, is adapted to the ODE-PDE under-actuated SASA system proposed herein. In doing so, the resultant closed-loop dynamics with the beam dynamics canceled by a feed-forward term are governed by a virtual dynamical system that converges exponentially to a stable manifold in the sense of spatial $\mathcal{L}_{2}$ norm. In the case with partial or no cancellation of the beam dynamics, finite-gain time-signal $\ell_{p}$ stability of the closed-loop system is proved by formulating the uncanceled beam dynamics as a disturbance term. From an end-to-end point of view, the controller actuates the flexible beam to achieve satellite slewing using the coupled ODE-PDE dynamics.

One of the major objectives of this paper is to experimentally demonstrate real-time closed-loop SASA (ODEPDE) attitude tracking control in the laboratory. In order to do so, it was necessary to discretize the infinitedimensional ODE-PDE plant dynamics and control law. Towards this end, Galerkin's method [12, 13, 15, 39] was used to form ODE systems from the PDE dynamics. The discretized control law was tested on the simulated plant dynamics before attempting implementation on the actual laboratory SASA testbed. The testbed consists of a one-degree-of-freedom cylinder (spacecraft bus) with flexible beams (solar arrays) symmetric about the cylinder's axis of rotation. Strain actuation in the beams is achieved by bonding piezoelectric actuators asymmetrically about the axis of the cylinder. 
The main contribution of this paper is the design of a new nonlinear ODE-PDE control law using the coupled structure in the dynamics to perform precision attitude control with detailed stability proofs. Notably, the proposed ODE-PDE control design captures all the modes of the flexible beam, thereby avoiding the spillover problem. Stable trajectory tracking via the nonlinear distributed control law is successfully demonstrated by analysis, numerical simulations, and experimental tests of the closed-loop system. The actuator model is validated by comparing the tip deflection of a composite cantilever beam (aluminum beam with surface-bonded PZT) with those obtained from simulations and experiments. The piezoelectric ceramic used in the actuators is lead zirconate titanate, which is abbreviated PZT based on its chemical formula: $\mathrm{Pb}\left[\mathrm{Zr}_{x} \mathrm{Ti}_{1-x}\right] \mathrm{O}_{3}, \quad(0 \leq x \leq 1)$. It is shown that the maximum controlled attitude rotation achieved by bending the solar array is constrained both by the inertia of the solar array and the bus, and by the maximum stress that can be applied to the solar array without compromising its structural integrity.

The paper is organized as follows. The kinematics, dynamics of the system, and actuator model of the composite beam (PZT and beam) are discussed in Section II Nonlinear ODE-PDE control law design and the associated stability analysis are presented in Section III Numerical implementation and results for trajectory tracking and slewing is given in Section IV Experimental results that validate the actuator model and real-time trajectory tracking of the bus rotation are given in Section $\mathrm{V}$ Concluding remarks are given in Section VI

\section{Dynamics of a 1-DOF Satellite with SASA}

The spacecraft with asymmetric SASAs is modeled as a cylinder with flexible appendages that are fixed symmetrically to the rotational axis of the cylinder as shown in Fig. 1] The dynamics of the system include planar rotation of the spacecraft bus and bending in the flexible composite beam due to strain-actuation. Our modeling approach uses explicit generalization of Lagrange's equations [15]. The equations of motion of the ODE-PDE system can also be derived by following the approach discussed in [40, 41]. Before proceeding to the Euler-Lagrange equations, we discuss briefly the system kinematics, physical properties of the composite beam, and the actuator model for strain-actuation using PZTs.

\section{II.A. Kinematics}

The spacecraft body is modeled as a cylinder of radius $r$, and the solar array is modeled as a composite beam of length $\ell$, with PZT actuators bonded on the beam surface. It is assumed that the beam does not undergo any longitudinal vibration. The coordinate systems used in the derivation of kinematics are shown in Fig. 1. In the $\left(x_{1}, y_{1}\right)$ coordinate system, the location of a point $P$ on an element $d x$ of the beam is given by $R_{P / A}=[x, \xi]$, where $\xi(x, t):[0, \ell] \times \mathbb{R}^{+} \rightarrow \mathbb{R}$ is the displacement due to bending in $y_{1}$ direction. The 


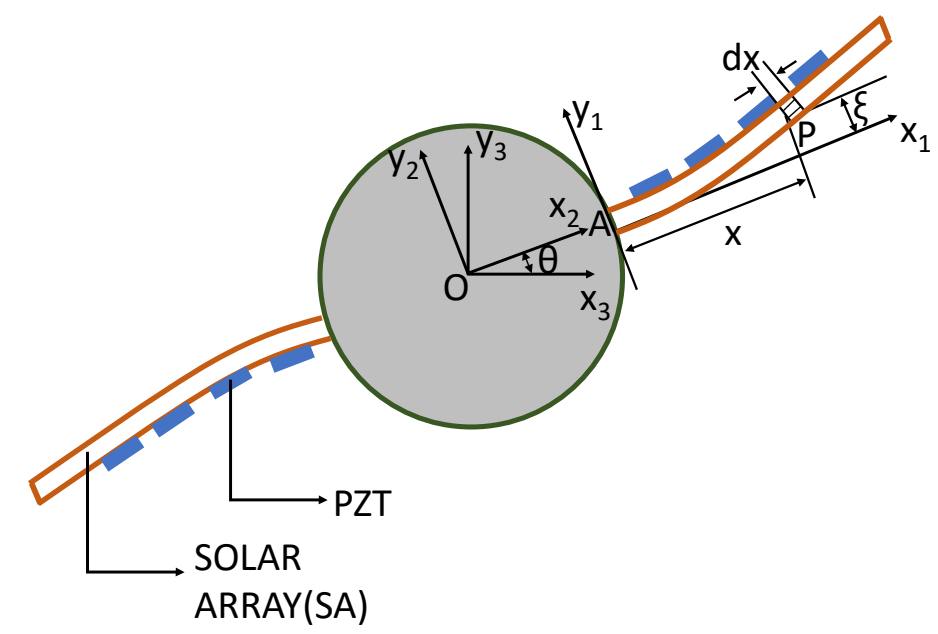

Figure 1: 1-DOF cylinder and flexible solar array model.

spacecraft body rotation angle about origin $O$ is $\theta(t)$, where $\theta(t): \mathbb{R}^{+} \rightarrow[-\pi, \pi]$. The position and velocity of $P$ with respect to origin of the spacecraft $O$ are given by Eqs. (1) and (2), respectively. These equations are used to compute $\xi$ using $x_{3}$ and $y_{3}$.

$$
\left[\begin{array}{l}
x_{3} \\
y_{3}
\end{array}\right]=\left[\begin{array}{cc}
\cos (\theta) & -\sin (\theta) \\
\sin (\theta) & \cos (\theta)
\end{array}\right]\left[\begin{array}{c}
r+x \\
\xi
\end{array}\right]
$$

The velocity kinematics are given by:

$$
\left[\begin{array}{c}
\dot{x}_{3} \\
\dot{y}_{3}
\end{array}\right]=\left[\begin{array}{cc}
-\sin (\theta) & -\cos (\theta) \\
\cos (\theta) & -\sin (\theta)
\end{array}\right]\left[\begin{array}{c}
(r+x) \dot{\theta}+\dot{\xi} \\
\xi \dot{\theta}
\end{array}\right]
$$

\section{II.B. Mass Per Unit Length and Total Rigidity of the Composite Beam}

The physical properties of the solar array, such as mass per unit length and location of the neutral axis that plays an important role in the evolution of the system dynamics, are functions of the spatial variable $x$ due to the composite nature of the beam. The mass per unit length of the composite beam is given in Eq. (3).

$$
m_{R}(x)=m_{b}+k(x) m_{p}, m_{b}=\rho_{b} A_{b}, m_{p}=\rho_{p} A_{p}
$$

where $\rho_{b}$ and $\rho_{p}$ denote the beam and the PZT densities, respectively. Also, $A_{b}$ and $A_{p}$ are the cross sectional areas of the beam and the PZT, respectively. Note that the function $k(x)=1$ at the locations where the PZT is bonded, and $k(x)=0$ otherwise.

$$
m_{R}(x)=m_{b}+k(x) m_{p}+\sum_{i=1}^{n_{v}} m_{v} \delta_{d}\left(x-\ell_{v_{i}}\right)
$$

$$
7 \text { of } 39
$$


For simulations and experiments described in this paper, we use Eq. (4) for the mass per unit length, which takes into account the effect of point masses placed on the beam for a particular sensing system we used. The point mass is $m_{v}, \delta_{d}$ is the Dirac delta function, $\ell_{v_{i}}$ is the distance to the $i$-th point mass on the beam from the root, and $n_{v}$ is the number of point masses.

The physical, structural, and geometric properties of the PZTs and the beam are different. As a result, the elastic neutral axis of the composite beam is offset from the geometric centroid. The distance between the top surface of the composite beam to the neutral axis $h_{n}$, as shown in Fig. 2a is given as:

$$
h_{n}=\frac{E_{p} t_{p}^{2}+E_{b} t_{b}\left(t_{b}+2 t_{p}\right)}{2\left(E_{p} t_{p}+E_{b} t_{b}\right)}
$$

where $E_{b}, I_{b}, t_{b}, E_{p}, I_{p}$, and $t_{p}$ are the Young's modulus, area moment of inertia about neutral axis, and thickness of the beam and the PZT, respectively.

The total beam rigidity $E I_{t}$ at the locations where PZTs are bonded, with area moment of inertia calculated about the new neutral axis, is given by:

$$
\begin{gathered}
E I_{t}=E_{b} I_{b}+E_{p} I_{p} \\
\text { where } I_{b}=\frac{w t_{b}^{3}}{12}+w t_{b}\left(t_{p}+\frac{t_{b}}{2}-h_{n}\right)^{2}, \quad I_{p}=\frac{w t_{p}^{3}}{12}+w t_{p}\left(h_{n}-\frac{t_{p}}{2}\right)^{2}
\end{gathered}
$$

The total beam rigidity $E I_{b}$ at locations with $k(x)=0$ (where no PZT actuator is attached) is given by following equation:

$$
E I_{b}=\frac{E_{b} w t_{b}^{3}}{12}
$$

In the dynamic model, we use the variable $E I(x)$ for rigidity of the beam, which is a function of spatial position $x$.

\section{II.C. Actuator Model}

Piezoelectric materials undergo uniform strain when an electrical potential difference is applied across their electrodes [42]. Due to this and other desirable properties, PZT actuators are commonly used in design of intelligent structures, active vibration control, and strain-actuated beams [22, 43 47]. PZT patches can be bonded to the surface of a solar array, or embedded within the solar array substructure [44, 48]. References [44, 48 discuss actuator models for the SASA configurations described above, with and without perfect bonding. Herein, a quasi-static actuator model based on the surface bonded PZT model [46] is assumed, including some needed error corrections in the final model.

The following actuator model provides the mathematical framework for modeling moments applied to the beam due to a voltage $V$ applied across the PZT bonded to the beam surface. In deriving the model, it 


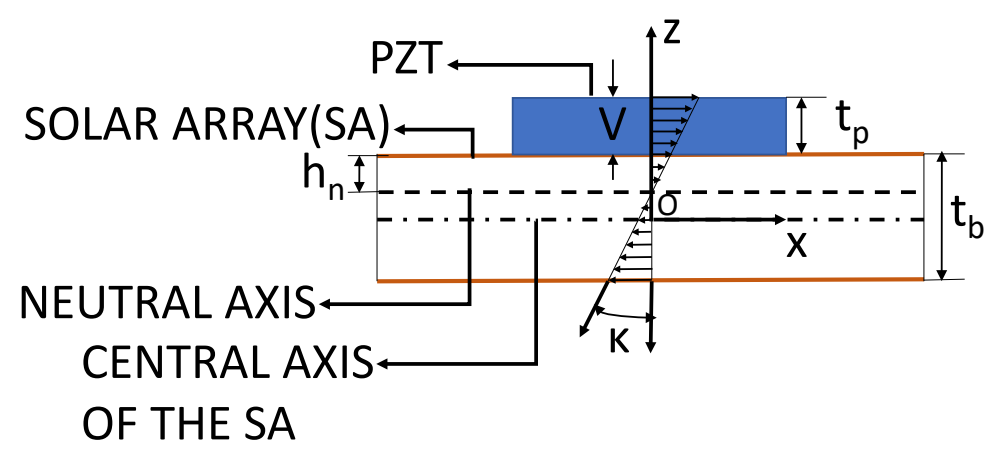

(a) Strain distribution.
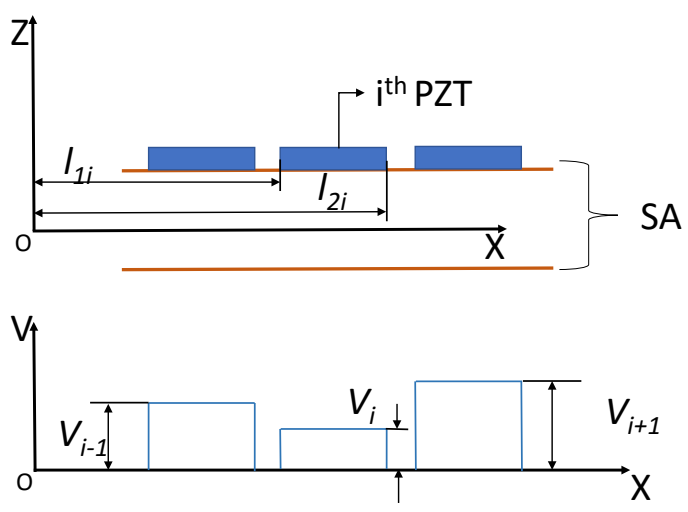

(b) Distributed Piezo.

Figure 2: Distributed Piezo and and strain distribution across the composite beam.

is assumed that the bonding between the PZT and the beam is perfect, and that the composite beam has constant width ' $w$ '. Here, the PZT is bonded only on the top surface and the strain distribution ' $\epsilon$ ' along the cross section of the composite beam is assumed to be linear (see Fig. 2a):

$$
\epsilon=\kappa z+\epsilon_{0}
$$

where $\kappa$ is the slope of the strain distribution due to bending, and $\epsilon_{0}$ is the beam extension due to the neutral axis offset. Force and moment equilibria are used to evaluate the expressions for $\kappa$ and $\epsilon_{0}$ (for detailed derivation, see [49]). The static model derived here is used in the dynamic model, assuming that the process is quasi-static in nature. In the following, $\epsilon_{p}$ is the strain of an unconstrained PZT when a voltage ' $V$ ' is applied across it. This strain is given by: $\epsilon_{p}=\frac{d_{z x} V}{t_{p}}$, [46]. The piezoelectric coefficient $d_{z x}$ is the ratio of strain in the $x$-direction when an electric field is applied across the PZT in the $z$-direction.

$$
\kappa=\frac{6 E_{b} E_{p} \epsilon_{p} t_{b} t_{p}\left(t_{b}+t_{p}\right)}{E_{b}^{2} t_{b}^{4}+4 E_{b} E_{p} t_{b}^{3} t_{p}+6 E_{b} E_{p} t_{b}^{2} t_{p}^{2}+4 E_{b} E_{p} t_{b} t_{p}^{3}+E_{p}^{2} t_{p}^{4}}
$$




$$
\epsilon_{0}=\frac{\epsilon_{p} E_{p} t_{p}\left(E_{p} t_{p}^{3}+E_{b} t_{b}^{3}\right)}{E_{b}^{2} t_{b}^{4}+4 E_{b} E_{p} t_{b}^{3} t_{p}+6 E_{b} E_{p} t_{b}^{2} t_{p}^{2}+4 E_{b} E_{p} t_{b} t_{p}^{3}+E_{p}^{2} t_{p}^{4}}
$$

The moment in the beam, due to the uniform PZT strain produced by the applied voltage $V$, is given by

$$
\begin{aligned}
M_{b} & =E_{b} I_{b} \kappa=c V, \\
\text { where } c & =\frac{6 E_{b}^{2} I_{b} E_{p} d_{z x} t_{b}\left(t_{b}+t_{p}\right)}{E_{b}^{2} t_{b}^{4}+4 E_{b} E_{p} t_{b}^{3} t_{p}+6 E_{b} E_{p} t_{b}^{2} t_{p}^{2}+4 E_{b} E_{p} t_{b} t_{p}^{3}+E_{p}^{2} t_{p}^{4}}
\end{aligned}
$$

The moment is proportional to the voltage applied across the PZT. The constant $c$ depends on the geometric parameters, the structural properties of the PZT, and the elastic modulus of the composite beam. The actuator model is extended to a scenario with multiple PZTs using step functions (see Fig. 2b). The model with $n$ PZTs on the beam surface is given in Eq. 12, where $V_{i}$ is the voltage applied across the $i$ th PZT, $c_{i}$ is the proportionality constant corresponding to the structural and geometric properties of the $i$ th PZT, and $u(x)$ is the step function with unit amplitude. The lengths $l_{1 i}$ and $l_{2 i}$ are defined for $i$ th PZT as shown in Fig. 2b

$$
M_{b}=\sum_{i=1}^{n} c_{i} V_{i}\left(u\left(x-l_{1 i}\right)-u\left(x-l_{2 i}\right)\right)
$$

Equations (11) and 12 are used to compute voltage signals required to achieve attitude control from the control signal in the simulations and experiments.

\section{II.D. Dynamics}

The system dynamics include elastic bending of the composite beam, modeled using Euler-Bernoulli beam theory, and rotational motion, which is modeled as planar rigid-body spacecraft rotation. The dynamic model, prescribed by the coupled ODE-PDE system with fixed-root and free-end boundary conditions, uses a hybrid coordinate system integrating one rigid body with an infinite-dimensional system (also called as distributed parameter system). In deriving the model, we assume that the deflections due to bending are small and that the beam has no longitudinal vibration; the effect of $\epsilon_{0}$ in Eq. (10) is assumed to be negligible. The state of the system corresponding to beam deflection is $\xi(x, t)$, described by a continuous function of spatial radial position $x$ and time $t$.

\section{II.D.1. Euler-Lagrange Equations}

The system Lagrangian $L$ involves several quantities, including the spacecraft bus (cylinder) mass moment of inertia $J_{\theta}$, rotational kinetic energy of the spacecraft body $T_{s}(\mathrm{Eq} .13)$, beam kinetic energy assuming asymmetric strain-actuation $T_{b}$ (Eq. (14)), and elastic potential energy and axial stiffening of the beams due 
to centrifugal force from bus rotation $U$ (Eq. (15)).

$$
\begin{aligned}
T_{s} & =\frac{1}{2} J_{\theta} \dot{\theta}^{2} \\
T_{b} & =\int_{0}^{\ell} m_{R}\left[\dot{\theta}^{2}\left(r^{2}+x^{2}+\xi^{2}\right)+\dot{\xi}^{2}+2 r \dot{\xi} \dot{\theta}+2 r \dot{\theta}^{2} x+2 \dot{\xi} \dot{\theta} x\right] d x \\
U & =\int_{0}^{\ell}\left[E I\left(\dot{\xi}^{\prime \prime}\right)^{2}\right] d x+\int_{0}^{\ell}\left[P(\dot{\theta}, x)\left(\xi^{\prime}\right)^{2}\right] d x
\end{aligned}
$$

The Lagrangian of the ODE-PDE system is given as $L=T_{s}+T_{b}-U$. Note that ()$^{\prime}=\frac{\partial}{\partial x}()$, and $P(\dot{\theta}, x)=$ $\int_{r+x}^{r+\ell}\left[m_{R} \dot{\theta}^{2} s\right] d s=p(x) \dot{\theta}^{2}$ is the axial tension in the beam due to rotation, where $m_{R}$ is expressed as a function of $s=r+x$. The definition of the composite beam total rigidity EI is given in Section II.B The nonconservative work $W_{\mathrm{nc}}$ done due to the moment applied by strain actuation is given by:

$$
W_{\mathrm{nc}}=\int_{0}^{\ell} \frac{M_{b}(x, t)^{2}}{E_{b} I_{b}} d x
$$

where $M_{b}(x, t)$ is the internal beam moment (see Eq. (11) ) due to the strain produced by PZTs. The equations of motion are obtained by using the explicit generalization of Lagrange's equations for infinite-dimensional systems approach (see [15] for more details). The extended Hamilton's principle can be stated as follows, where $t_{0}$ and $t_{f}$ are the initial and final time values, respectively:

$$
\int_{t_{0}}^{t_{f}}\left(\delta L+\delta W_{\mathrm{nc}}\right) d t=0
$$

Applying integration by parts to the expanded variations in terms of the state variables and using boundary conditions, we obtain the following equations of motion in terms of the Lagrangian:

$$
\begin{gathered}
\frac{d}{d t}\left(\frac{\partial L}{\partial \dot{\theta}}\right)-\frac{\partial L}{\partial \theta}=0 \\
\frac{d}{d t}\left(\frac{\partial L}{\partial \dot{\xi}}\right)-\frac{\partial L}{\partial \xi}+\frac{\partial}{\partial x}\left(\frac{\partial L}{\partial \xi^{\prime}}\right)-\frac{\partial^{2}}{\partial x^{2}}\left(\frac{\partial L}{\partial \xi^{\prime \prime}}\right)=\frac{\partial^{2}}{\partial x^{2}}\left(M_{b}(x, t)\right)
\end{gathered}
$$

The structural damping in the system is modeled using the Kelvin-Voigt constant $\mu$. The equations of motion after substituting the Lagrangian are:

$$
\begin{array}{r}
\left(J_{\theta}+2 \int_{0}^{\ell}\left(m_{R}\left((x+r)^{2}+\xi^{2}\right)-p(x) \xi^{\prime 2}\right) d x\right) \ddot{\theta}+2 \int_{0}^{\ell} m_{R}(r+x) \ddot{\xi} d x \\
+\int_{0}^{\ell} 4 m_{R} \dot{\xi} \dot{\theta} d x-\int_{0}^{\ell} 4 \dot{\theta} p(x) \xi^{\prime} \dot{\xi}^{\prime} d x=0
\end{array}
$$




$$
2 m_{R}(r+x) \ddot{\theta}+2 m_{R} \ddot{\xi}-2 m_{R} \dot{\theta}^{2} \xi-2\left(\dot{\theta}^{2} p(x) \xi^{\prime}\right)^{\prime}+2\left(E I \xi^{\prime \prime}+\mu E I \dot{\xi}^{\prime \prime}\right)^{\prime \prime}=2\left(M_{b}(x, t)\right)^{\prime \prime}
$$

The beam boundary conditions, due to the fixed root and free end, are given as:

$$
\left.\xi(x, t)\right|_{x=0}=\left.\xi^{\prime}(x, t)\right|_{x=0}=0,\left.\left(E I \xi^{\prime \prime}+\mu E I \dot{\xi}^{\prime \prime}\right)\right|_{x=\ell}=0,\left.\left(E I \xi^{\prime \prime}+\mu E I \dot{\xi}^{\prime \prime}\right)^{\prime}\right|_{x=\ell}=0
$$

The physical and structural properties of the bus and the solar array constrain the maximum slew angle that can be achieved by SASA. The linearized dynamics of Eqs. 20) and (21) are given as:

$$
\begin{aligned}
& \int_{0}^{\ell}\left(J_{\theta}+2 m_{R}(x+r)^{2}\right) d x \ddot{\theta}+\int_{0}^{\ell} 2 m_{R}(x+r) \ddot{\xi} d x=0 \\
& \int_{0}^{\ell} 2 m_{R}(x+r) d x \ddot{\theta}+\int_{0}^{\ell} 2 m_{R} \ddot{\xi} d x+\int_{0}^{\ell} 2\left(E I \xi^{\prime \prime}\right)^{\prime \prime} d x=\int_{0}^{\ell} 2\left(M_{b}(x, t)\right)^{\prime \prime} d x
\end{aligned}
$$

The linearized dynamics, Eqs. (23) and (24), correspond to momentum equilibrium about the axis of rotation and force equilibrium of an element $d x$ of the solar array, respectively. It is evident from these equations that the bus angular acceleration is bounded due to the bus inertia, beam inertia, beam mass distribution, and the shear force term $\int_{0}^{\ell} 2\left(E I \xi^{\prime \prime}\right)^{\prime \prime} d x$. The shear force $\int_{0}^{\ell} 2\left(E I \xi^{\prime \prime}\right)^{\prime \prime} d x$ across the solar array and the input strain actuation $\int_{0}^{\ell} 2\left(M_{b}(x, t)\right)^{\prime \prime} d x$ are bounded due to the structural constraints on the beam. The bus angular rotation is bounded, because a finite definite integral of a bounded continuous function is bounded.

\section{Nonlinear ODE-PDE Control Design of SASA}

The equations of motion in Eqs. 20) and (21) are re-written in Section III.A followed by a matrix form that is typical for robot system dynamic models. Equations 200 and 21) are expressed in the standard Euler-Lagrangian matrix form in Eq. 25 by applying integration by parts to the term $\int_{0}^{\ell} \dot{\theta} p(x) \xi^{\prime} \dot{\xi}^{\prime} d x$ in Eq. 20].

\section{III.A. Matrix Form of Euler-Lagrangian System}

$$
\begin{gathered}
\int_{0}^{\ell} M_{s}(x)\left[\begin{array}{l}
\ddot{\theta} \\
\ddot{\xi}
\end{array}\right] d x+\int_{0}^{\ell} C_{s}(x)\left[\begin{array}{l}
\dot{\theta} \\
\dot{\xi}
\end{array}\right] d x+\left[\begin{array}{c}
0 \\
S_{s}
\end{array}\right]=\left[\begin{array}{c}
0 \\
2 \int_{0}^{\ell}\left(M_{b}(x, t)\right)^{\prime \prime} d x
\end{array}\right] \\
M_{s}(x, t)=\left[\begin{array}{cc}
m_{11}(\xi) & m_{12} \\
m_{12} & m_{22}
\end{array}\right]=\left[\begin{array}{cc}
\left(J_{\theta} / \ell+2\left(m_{R}\left((x+r)^{2}+\xi^{2}\right)-p(x) \xi^{\prime 2}\right)\right) & 2 m_{R}(x+r) \\
2 m_{R}(x+r) & 2 m_{R}
\end{array}\right]
\end{gathered}
$$




$$
\begin{gathered}
C_{s}(x, t)=\left[\begin{array}{cc}
c_{11}(\xi, \dot{\xi}) & c_{12}(\xi, \dot{\theta}) \\
-c_{12}(\xi, \dot{\theta}) & 0
\end{array}\right]=\left[\begin{array}{cc}
2\left(m_{R} \xi \dot{\xi}-p(x) \xi^{\prime} \dot{\xi}^{\prime}\right) & 2\left(m_{R} \dot{\xi}+\left(p(x) \xi^{\prime}\right)^{\prime} \dot{\theta}\right) \\
-2\left(m_{R} \dot{\theta} \dot{\theta}+\left(p(x) \xi^{\prime}\right)^{\prime} \dot{\theta}\right) & 0
\end{array}\right] \\
S_{s}(x, t)=2 \int_{0}^{\ell}\left(E I \xi^{\prime \prime}+\mu E I \dot{\xi}^{\prime \prime}\right)^{\prime \prime} d x
\end{gathered}
$$

It is important to note that $c_{11}=\frac{\dot{m}_{11}}{2}$ and $\left(\dot{M}_{s}-2 C_{s}\right)$ is skew-symmetric. This skew-symmetric property is exploited in the stability proof of the main control law. Also, the boundary conditions in Eq. 22] still apply.

\section{III.B. Preliminaries}

Definition 1. (Spatial $\mathcal{L}_{2}$-norm) Given the spatial domain $[0, \ell]$ with $\ell>0$, let the standard Hilbert space $\mathcal{Z}=\mathcal{L}_{2}\left([0, \ell], \mathbb{R}^{n}\right)$ be equipped with inner product $\left\langle z_{1}, z_{2}\right\rangle_{\mathcal{Z}}=\int_{0}^{\ell} z_{1}^{T} z_{2} d x$ and induced norm $\|z\|_{\mathcal{Z}}=$ $\sqrt{\int_{0}^{\ell} z^{T} z d x}$ for all spatial functions $z, z_{1}, z_{2}:[0, \ell] \mapsto \mathbb{R}^{n}$ in $\mathcal{Z}$.

Definition 2. (Temporal $\ell_{p}$-norm) Given the time signal $q(t) \in \mathbb{R}^{n} \forall t \geq 0$ and arbitrary $s \in[0, \infty)$, let $(q(t))_{s}$ be the truncation of $q(t)$ where $(q(t))_{s}=q(t)$ for $t \in[0, s]$, and $(q(t))_{s}=0$ otherwise. Let the truncated $\ell_{p}$ signal norm be $\left\|(q)_{s}\right\|_{\ell_{p}} \triangleq\left(\int_{0}^{s}\|q(t)\|^{p} d t\right)^{\frac{1}{p}}<\infty$ for $p \in[1, \infty)$, and $\left\|(q)_{s}\right\|_{\ell_{\infty}} \triangleq \sup _{t \geq 0}\left\|(q(t))_{s}\right\|<\infty$ for $p=\infty$, where $\|\cdot\|$ is any vector norm.

Definition 3. (Spatiotemporal norms) Given $q(x, t) \in \mathbb{R}^{n} \forall x \in[0, \ell]$ and $t \geq 0$, let $\mathcal{Q} \triangleq \ell_{p}\left(\mathbb{R}^{+}, \mathcal{L}_{2}\left([0, \ell], \mathbb{R}^{n}\right)\right.$ ) denote the Hilbert space with induced spatial $\mathcal{L}_{2}$ norm $\|q(t)\|_{\mathcal{L}_{2}} \triangleq \sqrt{\int_{0}^{\ell} q(x, t)^{T} q(x, t) d x}$ and truncated $\ell_{p}$ signal norm $\|q\|_{\mathcal{Q}} \triangleq\left\|\left(\|q(t)\|_{\mathcal{L}_{2}}\right)_{s}\right\|_{\ell_{p}}$.

In this paper, there are two generalized coordinates $\theta$ and $\xi$ such that $q(x, t) \triangleq[\theta(t), \xi(x, t)]^{T} \in \mathbb{R}^{2}$ where $\theta: \mathbb{R}^{+} \mapsto \mathbb{R}$ and $\xi:[0, \ell] \times \mathbb{R}^{+} \mapsto \mathbb{R}$.

\section{III.C. Properties of the Underactuated Hybrid System}

\section{III.C.1. Kinetic Symmetry}

The dynamics in Eq. 25] possesses kinetic symmetry [36] with respect to the spacecraft attitude $\theta$, as the inertia matrix $M_{s}(x)$ per unit length is independent of $\theta$. The kinetic symmetry with respect to $\theta$ in the 
absence of gravitational effects leads to symmetry in mechanics, satisfying Eq. 29.

$$
\begin{aligned}
& \frac{\partial K}{\partial \theta}=\frac{\partial L}{\partial \theta}=0 \\
& z_{1}=\frac{\partial L}{\partial \dot{\theta}}=\int_{0}^{\ell}\left(m_{11} \dot{\theta}+m_{12} \dot{\xi}\right) d x, \quad \dot{z}_{1}=\frac{d}{d t} \frac{\partial L}{\partial \dot{\theta}}=\frac{\partial L}{\partial \theta}=0
\end{aligned}
$$

The above is true because the corresponding Lagrangian $L$ is independent of $\theta$. Note that the first generalized angular momentum for the variable $\theta$, which is denoted by $z_{1}$, is given in Eq. 30.

\section{III.C.2. Positive Definiteness and Uniform Boundedness of the Inertia Matrix}

The positive definiteness and uniform bounds of the inertia matrix are used in the Lyapunov analysis of the closed-loop system to prove exponential stability. The inertia matrix here, unlike rigid-body robot dynamics, has axial stiffening component $-p(x) \xi^{2}$ in the $m_{11}$ term due to the centrifugal force on the solar array, which can make the inertia matrix non-positive definite. The effect of axial stiffing on the multibody dynamics is studied in [17, 50. Using the Sylvester's criterion, we obtain the inequalities presented in Eq. (31) for the positive definiteness of the $M_{s}$ matrix.

$$
\begin{array}{r}
J_{\theta} / \ell+2\left(m_{R}\left((x+r)^{2}+\xi^{2}\right)-p(x) \xi^{\prime 2}\right)>0 \\
2 m_{R} J_{\theta} / \ell+4 m_{R}^{2} \xi^{2}-4 m_{R} p(x) \xi^{\prime 2}>0
\end{array}
$$

These inequalities are always satisfied due to the small deflection assumption of the beam. Assuming constant $m_{R}$, the inequalities in the Eq. 31] can be simplified to Eq. 32.

$$
2 \xi^{2}-\xi^{\prime 2}((\ell-x)(\ell+x+2 r))>-\frac{J_{\theta}}{\ell m_{R}}
$$

The maximum of $((\ell-x)(\ell+x+2 r))$ occurs at $x=0$. Equation 32 is satisfied if $2 \xi^{2}-\xi^{\prime 2}\left(\ell^{2}+2 \ell r\right)>-\frac{J_{\theta}}{\ell m_{R}}$. This is used to define a domain of permissible displacement and strain envelope as $2 \xi^{2}+\frac{J_{\theta}}{\ell m_{R}}>\xi^{\prime 2}\left(\ell^{2}+2 \ell r\right)$. Let the bounds of the inertia matrix be given by using the spatial $\mathcal{L}_{2}$ norm as follows:

$$
0<\sigma_{1}\|q(t)\|_{\mathcal{L}_{2}}^{2} \leq \int_{0}^{\ell} q^{T} M_{s}(x, t) q d x \leq \sigma_{2}\|q(t)\|_{\mathcal{L}_{2}}^{2} ; \text { where } q(t)=(\theta, \xi)^{T}
$$

The bounds are not uniform because they depend on the state $\xi$, if $\xi \rightarrow \infty$ both $\sigma_{1}$ and $\sigma_{2}$ grow unbounded. In the configuration space of the system, $\xi(x)$ at any time $t$ is a function of the moment applied at that location and $\xi \leq \xi_{\max }$, where $\xi_{\max }$ is the maximum strain that can be achieved before violating structural constraints of the beam. For the Euler-Bernoulli beam considered, using the strain-moment relationship for symmetric bending $\xi_{\max }=\frac{h M_{\max }}{E I}$, where the thickness of the beam is $2 h, M_{\max }$ is the maximum moment 
that can be applied at a point, $E$ is the Young's modulus and $I$ is the moment of inertia respectively. Therefore, we can always find $\sigma_{1}$ and $\sigma_{2}$, which are uniform bounds on the inertia matrix $M_{s}$.

\section{III.D. Nonlinear ODE-PDE Control Law}

The control objective is to ensure that the bus rotation angle $\theta(t) \in C^{2}([0, \infty))$ tracks a desired attitude trajectory $\theta_{d}(t) \in C^{2}([0, \infty))$. The nonlinear control law is developed using the properties discussed in Section III.C To track a desired bus rotation trajectory $\theta_{d}$, a reference rotation velocity signal, as shown in Eq. (34), is computed by shifting the rotation velocity with the position error term in which $\lambda_{\theta}$ is a positive value. The reference rotation acceleration is obtained by taking the time derivative of the Eq. (34):

$$
\begin{aligned}
& \dot{\theta}_{r}(t)=\dot{\theta}_{d}(t)-\lambda_{\theta}\left(\theta-\theta_{d}(t)\right) \\
& \ddot{\theta}_{r}(t)=\ddot{\theta}_{d}(t)-\lambda_{\theta}\left(\dot{\theta}-\dot{\theta}_{d}(t)\right)
\end{aligned}
$$

The reference beam deflection signal $\xi_{r}$ is computed by integrating Eq. (36). The reference signal $\xi_{r}$ quantifies the $\theta$ dynamics for the reference signal $\theta_{r}$ that is augmented with the error feedback term $K_{\theta}\left(\dot{\theta}-\dot{\theta}_{r}\right)$, where $K_{\theta}$ is a positive feedback gain.

$$
m_{12} \ddot{\xi}_{r}+m_{11} \ddot{\theta}_{r}+c_{11} \dot{\theta}_{r}+c_{12} \dot{\xi}_{r}=K_{\theta}\left(\dot{\theta}-\dot{\theta}_{r}\right)
$$

Using Eqs. 34, 35, and (36), and variables $s_{\theta}=\dot{\theta}-\dot{\theta}_{r}$ and $s_{\xi}=\dot{\xi}-\dot{\xi}_{r}$, the control law is defined in Eq. [37, where $\tau=\left(M_{b}(x, t)\right)^{\prime \prime}$.

$$
\tau=m_{12} \ddot{\theta}_{r}+m_{22} \ddot{\xi}_{r}-c_{12} \dot{\theta}_{r}+2\left(\delta E I \xi^{\prime \prime}+\mu E I \delta \dot{\xi}^{\prime \prime}\right)^{\prime \prime}-K_{\xi} s_{\xi}
$$

The closed-loop system with this controller is given in Eq. [39), where $\delta$ is a constant $\in[0,1], K_{\xi}$ is a positive feedback gain and $q_{s}(x, t)=\left[\begin{array}{c}s_{\theta} \\ s_{\xi}\end{array}\right]$.

$$
\begin{gathered}
K_{s}=\left[\begin{array}{cc}
K_{\theta} & 0 \\
0 & K_{\xi}
\end{array}\right] ; \quad D\left(\delta, \xi^{\prime \prime}, \dot{\xi}^{\prime \prime}\right)=\left[\begin{array}{c}
0 \\
(1-\delta)\left(2 E I \xi^{\prime \prime}+\mu 2 E I \dot{\xi}^{\prime \prime}\right)
\end{array}\right] \\
\int_{0}^{\ell}\left(M_{s} \dot{q}_{s}+C_{s} q_{s}+D\left(\delta, \xi^{\prime \prime}, \dot{\xi}^{\prime \prime}\right)+K_{s} q_{s}\right) d x=0
\end{gathered}
$$

Notice that the parameter $\delta$ defines an auxiliary control input in the following sense. If $\delta=1$, the beam bending stiffness term $\left(S_{s}(x, t)\right.$ in Eq. 280$)$ is completely nulled (e.g., via feed-forward control), and if $\delta=0$, the beam bending dynamics are natural (uncompensated). For $\delta=1$, exponential stability of the closed-loop 
system is given in Theorem 1] and the robust stability against a bounded disturbance term is discussed in Theorem 2

Theorem 1. The closed-loop system, given in Eq. (40), with the control law Eqs. (34 37) and $\delta=1$, is globally exponentially stable in the sense of spatial $\mathcal{L}_{2}$ norm, defined in Definition 1 , assuming the feedback gains $K_{\xi}, K_{\delta}>0$ and the inertia matrix $M_{s}$ is positive definite by Eq. (31) and uniformly bounded as in Eq. (33).

$$
\int_{0}^{\ell}\left(M_{s} \dot{q}_{s}+C_{s} q_{s}+K_{s} q_{s}\right) d x=0
$$

Proof. The inertia matrix $M_{s}$, due to the properties discussed in Sections III.A and III.C.2, is used to compute a spatial norm Eq. (41) for a Lyapunov-like stability analysis [28]:

$$
V_{L}=\frac{1}{2} \int_{0}^{\ell} q_{s}^{T} M_{s}(x, t) q_{s} d x
$$

The time-derivative of $V_{L}$ is given as follows:

$$
\dot{V}_{L}=\int_{0}^{\ell} q_{s}^{T} M_{s} \dot{q}_{s} d x+\frac{1}{2} \int_{0}^{\ell} q_{s}^{T} \dot{M}_{s} q_{s} d x ; \text { where } \dot{M}_{s}=\left[\begin{array}{cc}
\dot{m}_{11}(\xi) & 0 \\
0 & 0
\end{array}\right]
$$

Using $\frac{\dot{m}_{11}(\xi)}{2}=c_{11}$, and adding and subtracting $c_{12}(\xi, \dot{\theta}) s_{\xi} s_{\theta}$, it can be simplified to Eq. 43 .

$$
\dot{V}_{L}=-\int_{0}^{\ell} q_{s}^{T} K_{s} q_{s} \leq-\frac{2 K}{\sigma_{2}} V_{L}
$$

In the above inequality, we used the uniform bounds on the inertia matrix as given in Eq. 33 , and $K=$ $\min \left\{K_{\theta}, K_{\xi}\right\}>0$. Also, note that the skew-symmetric property of $\dot{M}_{s}-2 C_{s}$ is used. Using the Comparison Lemma [51], and the bounds on the inertia matrix, we obtain:

$$
\begin{aligned}
V_{L}(t) & \leq \exp \left(-\frac{2 K}{\sigma_{2}} t\right) V_{L}(0) \\
\left\|\left[\begin{array}{c}
s_{\theta}(t) \\
s_{\xi}(x, t)
\end{array}\right]\right\|_{\mathcal{L}_{2}}^{2} & \leq \frac{\sigma_{2}}{\sigma_{1}} \exp \left(-\frac{2 K}{\sigma_{2}} t\right)\left\|\left[\begin{array}{c}
s_{\theta}(0) \\
s_{\xi}(x, 0)
\end{array}\right]\right\|_{\mathcal{L}_{2}}^{2} \\
\left\|\left[\begin{array}{c}
s_{\theta}(t) \\
s_{\xi}(x, t)
\end{array}\right]\right\|_{\mathcal{L}_{2}} & \leq \sqrt{\frac{\sigma_{2}}{\sigma_{1}}} \exp \left(-\frac{K}{\sigma_{2}} t\right)\left\|\left[\begin{array}{c}
s_{\theta}(0) \\
s_{\xi}(x, 0)
\end{array}\right]\right\|_{\mathcal{L}_{2}}
\end{aligned}
$$

Therefore, the system in Eq. 40 converges exponentially to the manifold $s_{\theta}=0$ and $s_{\xi}=0$ for a positive definite gain matrix in the sense of $\mathcal{L}_{2}, \int_{0}^{\ell}\left(\dot{\theta}-\dot{\theta}_{r}\right)^{2} d x \rightarrow 0$ and $\int_{0}^{\ell}\left(\dot{\xi}-\dot{\xi}_{r}\right)^{2} d x \rightarrow 0$, implying $\dot{\theta} \rightarrow \dot{\theta}_{r}$ and $\dot{\xi} \rightarrow \dot{\xi}_{r}$. To prove $\theta \rightarrow \theta_{d}$, we need to subtract $\dot{\theta}$ from both sides of the Eq. 34 to obtain $\dot{\theta}_{r}(t)-\dot{\theta}^{3}=\dot{\theta}_{d}(t)-$ $\dot{\theta}-\lambda_{\theta}\left(\theta-\theta_{d}(t)\right)$, and use the fact that $\left(\dot{\theta}-\dot{\theta}_{r}\right) \rightarrow 0$ to form the first-order ODE, $\dot{\theta}-\dot{\theta}_{d}(t)=-\lambda_{\theta}\left(\theta-\theta_{d}(t)\right)$. This equation becomes $\dot{e}=-\lambda e$ with $e=\theta-\theta_{d}(t)$, which implies $e(t)=e(0) \exp \left[-\lambda_{\theta} t\right]$ for any initial 
tracking error condition $e(0) \in \mathbb{R}$. Thus $\theta \rightarrow \theta_{d}$ holds globally with an exponential rate of convergence $\lambda_{\theta}>0$

If $\delta \neq 1$, the beam dynamics term $D\left(\delta, \xi^{\prime \prime}, \dot{\xi}^{\prime \prime}\right)$ is regarded as a bounded disturbance at the input of the closed-loop system in Eq. 40, where $\delta \in[0,1)$. The closed-loop dynamics in Eq. (39) can be written as the following equation:

$$
\int_{0}^{\ell}\left(M_{s} \dot{q}_{s}+C_{s} q_{s}+K_{s} q_{s}\right) d x=-\int_{0}^{\ell} D\left(\delta, \xi^{\prime \prime}, \dot{\xi}^{\prime \prime}\right) d x
$$

In order to satisfy the structural constraints and the Euler-Bernoulli beam assumption, the term $D\left(\delta, \xi^{\prime \prime}, \dot{\xi}^{\prime \prime}\right)$ must be bounded in the context of the Definitions 1.3 This situation can be generalized to a case when the closed-loop system in Eq. 40 has a bounded perturbation $\int_{0}^{\ell} d(x, t) d x$ at the input, as defined in Theorem 2 The stability of the closed-loop system in Eq. 45 is essential for tracking a trajectory when $\delta \neq 1$ with a bounded tracking error. In the Theorem 2 , we prove the finite-gain $\ell_{p}$ stability of the closed-loop system in Eq. 40 in the sense of spatial $\mathcal{L}_{2}$ norm by approximating $-\int_{0}^{\ell} D\left(\delta, \xi^{\prime \prime}, \dot{\xi}^{\prime \prime}\right) d x$ in the Eq. 45 as a general bounded disturbance term $\int_{0}^{\ell} d(x, t) d x$ as an the input to the closed-loop system in Eq. 40 .

Theorem 2. The closed-loop system, given in Eq. (40), with a bounded perturbation at the input of the form $\int_{0}^{\ell} d(x, t) d x=\int_{0}^{\ell}\left(\left[\begin{array}{c}\delta_{\theta} \\ \delta_{\xi}\end{array}\right]+\left[\begin{array}{cc}\gamma_{\theta} & 0 \\ 0 & \gamma_{\xi}\end{array}\right]\left[\begin{array}{l}s_{\theta} \\ s_{\xi}\end{array}\right]\right) d x$ exponentially converges to the error ball, $\lim _{t \rightarrow \infty}\left\|\left[\begin{array}{c}s_{\theta}(t) \\ s_{\xi}(x, t)\end{array}\right]\right\| \mathcal{L}_{\mathcal{L}_{2}} \rightarrow$ $\frac{\sigma_{2} \gamma_{\infty}}{\sigma_{1} K}$, assuming that the inertia matrix $M_{s}$ is positive definite by Eq. (31) and uniformly bounded by Eq. (33). Also, it is assumed that the nonvanishing perturbation term at the origin, $\delta_{\mathcal{L}_{2}}(t)=\left\|\left[\begin{array}{c}\delta_{\theta} \\ \delta_{\xi}\end{array}\right]\right\|_{\mathcal{L}_{2}}$ satisfies the bound $\sup _{t \geq 0}\left|\delta_{\mathcal{L}_{2}}(t)\right| \leq \gamma_{\infty}$. Note that the functions $\gamma_{\theta}, \gamma_{\xi}: \mathbb{R} \rightarrow \mathbb{R}$ are nonnegative and continuous $\forall t \geq 0$ and $\gamma_{\infty}$ is a positive constant. Furthermore, Eq. (40) is finite-gain $\ell_{p}$ stable $\forall p \in[1, \infty]$ in the sense of spatial $\mathcal{L}_{2}$ norm, given that the $\ell_{p}$ time norm of $\delta_{\mathcal{L}_{2}}(t)$ is bounded, as in $\left\|\left(\delta_{\mathcal{L}_{2}}(t)\right)_{s}\right\|_{\ell_{p}} \leq \gamma_{p}$, where $\gamma_{p}$ is a positive constant.

Proof. We proceed to take the $V_{L}$ as in Eq. 41, $V_{L}=\frac{1}{2} \int_{0}^{\ell} q_{s}^{T} M_{s}(x, t) q_{s} d x$. Using the skew-symmetric property of $\dot{M}_{s}-2 C_{s}$, its time-derivative simplifies to the following Eq. 46 .

$$
\dot{V}_{L} \leq-\frac{2}{\sigma_{2}} \min \left\{K_{\theta}, K_{\xi}\right\} V_{L}+\frac{2}{\sigma_{1}} \max \left\{\gamma_{\theta}, \gamma_{\xi}\right\} V_{L}+\int_{0}^{\ell}\left(s_{\theta} \delta_{\theta}+s_{\xi} \delta_{\xi}\right) d x
$$

Let $K \triangleq\left(\min \left\{K_{\theta}, K_{\xi}\right\}-\frac{\sigma_{2}}{\sigma_{1}} \max \left\{\gamma_{\theta}, \gamma_{\xi}\right\}\right)$. For sufficiently large $K_{\theta}, K_{\xi}>0$, we can prove $K>0, \forall t \geq 0$. Using the Cauchy-Schwarz inequality,

$$
\begin{aligned}
\dot{V}_{L} & \leq-\frac{2 K}{\sigma_{2}} V_{L}+\left\|\left[\begin{array}{c}
s_{\theta}(t) \\
s_{\xi}(x, t)
\end{array}\right]\right\|_{\mathcal{L}_{2}}\left\|\left[\begin{array}{c}
\delta_{\theta} \\
\delta_{\xi}
\end{array}\right]\right\|_{\mathcal{L}_{2}} \\
& \leq-\frac{2 K}{\sigma_{2}} V_{L}+\sqrt{\frac{2 V_{L}}{\sigma_{1}}} \delta_{\mathcal{L}_{2}}(t)
\end{aligned}
$$


Using the transformation $W=\sqrt{V_{L}}$, followed by application of the Comparison Lemma [51] and the uniform bounds on the inertia matrix Eq. 33 , we get the following inequality in terms of spatial $\mathcal{L}_{2}$ norm of states.

$$
\left\|\left[\begin{array}{c}
s_{\theta}(t) \\
s_{\xi}(x, t)
\end{array}\right]\right\|_{\mathcal{L}_{2}} \leq \sqrt{\frac{\sigma_{2}}{\sigma_{1}}} \exp \left(-\frac{K t}{\sigma_{2}}\right)\left\|\left[\begin{array}{c}
s_{\theta}(0) \\
s_{\xi}(x, 0)
\end{array}\right]\right\|_{\mathcal{L}_{2}}+\frac{1}{\sigma_{1}} \int_{0}^{t}\left(\delta_{\mathcal{L}_{2}}(\tau) \exp \left(-\frac{K(t-\tau)}{\sigma_{2}}\right)\right) d \tau
$$

Given that $\sup _{\tau \geq 0}\left|\delta_{\mathcal{L}_{2}}(\tau)\right| \leq \gamma_{\infty}$, moving it to outside the integral and expanding the integral, we get the following inequality.

$$
\left\|\left[\begin{array}{c}
s_{\theta}(t) \\
s_{\xi}(x, t)
\end{array}\right]\right\|_{\mathcal{L}_{2}} \leq \sqrt{\frac{\sigma_{2}}{\sigma_{1}}} \exp \left(-\frac{K t}{\sigma_{2}}\right)\left\|\left[\begin{array}{c}
s_{\theta}(0) \\
s_{\xi}(x, 0)
\end{array}\right]\right\|_{\mathcal{L}_{2}}+\frac{\sigma_{2} \gamma_{\infty}}{\sigma_{1} K}\left(1-\exp \left(-\frac{K t}{\sigma_{2}}\right)\right)
$$

By taking a limit $t \rightarrow \infty$ on both sides of Eq. 50 , we get the bound, $\lim _{t \rightarrow \infty}\left\|\left[\begin{array}{c}s_{\theta}(t) \\ s_{\xi}(x, t)\end{array}\right]\right\|_{\mathcal{L}_{2}} \rightarrow \frac{\sigma_{2} \gamma_{\infty}}{\sigma_{1} K}$, on the spatial $\mathcal{L}_{2}$ norm of the states. Taking $\ell_{p}$ norm on both sides of the Eq. [49], with $q_{s}(x, t)=\left[s_{\theta}, s_{\xi}\right]$, we get the following inequality.

$$
\begin{aligned}
\left\|q_{s}\right\|_{\mathcal{Q}} & \leq \sqrt{\frac{\sigma_{2}}{\sigma_{1}}}\left\|q_{s}(x, 0)\right\|_{\mathcal{L}_{2}}\left\|\exp \left(-\frac{K t}{\sigma_{2}}\right)\right\|_{\ell_{p}}+\frac{1}{\sigma_{1}}\left\|\exp \left(-\frac{K t}{\sigma_{2}}\right)\right\|_{\ell_{1}}\left\|\delta_{\mathcal{L}_{2}}(t)\right\|_{\ell_{p}} \\
& \leq \sqrt{\frac{\sigma_{2}}{\sigma_{1}}}\left\|q_{s}(x, 0)\right\|_{\mathcal{L}_{2}} \zeta+\frac{\sigma_{2}}{K \sigma_{1}}\left\|\delta_{\mathcal{L}_{2}}(t)\right\|_{\ell_{p}} \\
& \leq \sqrt{\frac{\sigma_{2}}{\sigma_{1}}}\left\|q_{s}(x, 0)\right\|_{\mathcal{L}_{2}} \zeta+\frac{\sigma_{2} \gamma_{p}}{\sigma_{1} K}
\end{aligned}
$$

In Eq. $52, \zeta=1$ if $p=\infty$ and $\zeta=\frac{1}{\left(K p / \sigma_{2}\right)^{\frac{1}{p}}}$ if $p \in[1, \infty)$. Thus, the closed-loop system with bounded disturbance is shown to be finite-gain time-signal $\ell_{p}$ norm stable with $\ell_{p}$ and $\mathcal{L}_{2}$ norms defined in Definitions 1.3 using Theorem 5.1 in [51].

Remark. Theorem 1 offers global exponential stability in the spatial $\mathcal{L}_{2}$ norm sense under the implicit assumption that the underlying Euler-Bernoulli beam model remains valid globally; i.e., for all bending states with arbitrarily large strain energy. In practice, overloading physical beams causes plastic deformations which violate elastic beam model assumptions. To mitigate this issue, an auxiliary momentum management control system [10] is often needed to reduce strain levels before the onset of plasticity is allowed to occur.

In the control law in Eq. (37), not canceling the beam dynamics completely is a convenient way to add bounded disturbance at the input during simulations and experiments. The dynamics and the control laws discussed so far are all in PDE form. In the following section, we will discuss the implementation of the closed-loop system for simulations and experimental validation using finite-dimensional approximations of the system dynamics. The values of $\gamma_{\xi}$ and $\gamma_{\theta}$ that can be overcome with a high gain depends on the actuator saturation limits. 


\section{Control Implementation and Simulation Results}

\section{IV.A. Galerkin Formulation}

The ODE-PDE model is discretized to obtain an ODE model using the Galerkin method [12, 15] for numerical simulations and experiments. The beam deflection is approximated as $\xi(x, t)=\phi^{T}(x) \eta(t)$, where $\phi(x)$ and $\eta(t)$ are continuously differentiable functions with respect to $x, t$ respectively. $\phi(x)$ are Galerkin functions. These functions comprise an approximation of the basis for the space of possible deflections of the flexible beam. The discretization is implemented by minimizing the weighted residual of the $\xi(x, t)$ dynamics (see Eq. (54)).

$$
\int_{0}^{\ell} \phi\left(m_{R}(r+x) \ddot{\theta}+m_{R} \ddot{\xi}-m_{R} \dot{\theta}^{2} \xi-\left(\dot{\theta}^{2} p(x) \xi^{\prime}\right)^{\prime}+\left(E I \xi^{\prime \prime}+\mu E I \dot{\xi}^{\prime \prime}\right)^{\prime \prime}-M_{b}^{\prime \prime}(x, t)\right) d x=0
$$

The Galerkin functions $\phi_{j}(x)$, where $j \in\{1,2,3,4\}$, in Eq. (55) are taken from [15], and are chosen to satisfy boundary conditions. We use four Galerkin functions $\phi=\left[\phi_{1}(x), \phi_{2}(x), \phi_{3}(x), \phi_{4}(x)\right]^{T}$, which describe dynamics sufficiently for tracking a sinusoidal attitude trajectory and slewing shown here, as the frequency of the trajectory chosen is well below the first mode of the composite beam.

$$
\phi_{j}(x)=1-\cos \left(\frac{j \pi x}{\ell}\right)+\frac{1}{2}(-1)^{j+1}\left(\frac{j \pi x}{\ell}\right)^{2}
$$

\section{IV.B. Dynamics in Galerkin Form}

The equations of motion in Galerkin form are given in Eq. [56). The matrices $[A],[B]$, and $[C]$ are used to define the mass matrix $\left[M_{g}\right]$ and the gyroscopic cross coupling terms in matrix $\left[C_{g}\right]$. The matrix $[E]$ corresponds to beam stiffness. The matrices in Eq. (59) are referred to as Galerkin matrices in this paper.

$$
\begin{aligned}
& {\left[\boldsymbol{M}_{\boldsymbol{g}}\right]\left[\begin{array}{l}
\ddot{\theta} \\
\ddot{\boldsymbol{\eta}}
\end{array}\right]+\left[\boldsymbol{C}_{\boldsymbol{g}}\right]\left[\begin{array}{l}
\dot{\theta} \\
\dot{\boldsymbol{\eta}}
\end{array}\right]+\left[\begin{array}{c}
0 \\
2[\boldsymbol{E}](\boldsymbol{\eta}+\mu \dot{\boldsymbol{\eta}})
\end{array}\right]=\left[\begin{array}{c}
0 \\
\int_{0}^{\ell} 2 \phi M_{b}^{\prime \prime} d x
\end{array}\right]} \\
& {\left[\boldsymbol{M}_{\boldsymbol{g}}\right]=\left[\begin{array}{ll}
m_{11_{g}} & m_{12_{g}} \\
m_{21_{g}} & m_{22_{g}}
\end{array}\right]=\left[\begin{array}{cc}
\left(J_{\theta}+2 \int_{0}^{\ell} m_{R}(x+r)^{2} d x+2 \boldsymbol{\eta}^{T}([\boldsymbol{A}]-[\boldsymbol{B}]) \boldsymbol{\eta}\right) & 2[\boldsymbol{C}] \\
2[\boldsymbol{C}]^{T} & 2[\boldsymbol{A}]
\end{array}\right]} \\
& {\left[\boldsymbol{C}_{\boldsymbol{g}}\right]=\left[\begin{array}{cc}
c_{11_{g}} & c_{12_{g}} \\
c_{21_{g}} & c_{22_{g}}
\end{array}\right]=\left[\begin{array}{cc}
2 \dot{\boldsymbol{\eta}}^{T}([\boldsymbol{A}]-[\boldsymbol{B}]) \boldsymbol{\eta} & 2 \boldsymbol{\eta}^{T}([\boldsymbol{A}]-[\boldsymbol{B}]) \dot{\theta} \\
-2([\boldsymbol{A}]-[\boldsymbol{B}]) \boldsymbol{\eta} \dot{\theta} & 0
\end{array}\right]}
\end{aligned}
$$




$$
\begin{aligned}
& {[\boldsymbol{A}]=\int_{0}^{\ell} m_{R} \boldsymbol{\phi} \boldsymbol{\phi}^{T} d x, \quad[\boldsymbol{B}]=\int_{0}^{\ell} p \boldsymbol{\phi}^{\prime} \boldsymbol{\phi}^{\prime T} d x,} \\
& {[\boldsymbol{C}]=\int_{0}^{\ell} m_{R}(x+r) \boldsymbol{\phi}^{T} d x, \quad[\boldsymbol{E}]=\int_{0}^{\ell} \boldsymbol{\phi}\left(E I \boldsymbol{\phi}^{\prime \prime T}\right)^{\prime \prime} d x}
\end{aligned}
$$

\section{IV.C. Computation of Voltage Signal from Control Signal}

The voltage signal, which is the input to the PZT actuator, is computed from the control signal in Galerkin form $\int_{0}^{\ell} 2 \phi M_{b}^{\prime \prime} d x$ during experiments as follows. For $n$ discrete PZT actuators bonded onto the solar array (see Fig. 2b, the moment produced can be modeled as described in Eq. 12, which is used in the following:

$$
\begin{aligned}
\tau & =\int_{0}^{\ell} \phi M_{b}^{\prime \prime} d x \\
& =\int_{0}^{\ell} \phi \sum_{i=1}^{n} c_{i} V_{i}(t)\left(u\left(x-l_{1 i}\right)-u\left(x-l_{2 i}\right)\right)^{\prime \prime} d x \\
& =\sum_{i=1}^{n} c_{i} V_{i}(t) \int_{0}^{\ell} \phi\left(u\left(x-l_{1 i}\right)-u\left(x-l_{2 i}\right)\right)^{\prime \prime} d x
\end{aligned}
$$

where the function $u$ is a step function, whose derivative is the Dirac delta function $\delta_{d}$. The term $\int_{0}^{\ell} \phi\left(u\left(x-l_{1 i}\right)-u\left(x-l_{1 i}\right)\right)^{\prime \prime} d x$ can be simplified to $\int_{0}^{\ell}-\phi^{\prime}\left(\delta_{d}\left(x-l_{1 i}\right)-\delta_{d}\left(x-l_{1 i}\right) d x\right.$ using integration by parts. Using the shifting property of the impulse function, we get Eq. 63):

$$
\tau=\sum_{i=1}^{n} c_{i} V_{i}(t)\left(\phi^{\prime}\left(l_{2 i}\right)-\phi^{\prime}\left(l_{1 i}\right)\right)
$$

With two PZT actuators on the beam, the voltage signal is computed using Eq. 63) as follows:

$$
\begin{gathered}
\boldsymbol{\Phi}\left[\begin{array}{l}
V_{1} \\
V_{2}
\end{array}\right]=\tau, \text { where } \boldsymbol{\Phi}=\left[\begin{array}{ll}
\phi_{1}^{\prime}\left(l_{21}\right)-\phi_{1}^{\prime}\left(l_{11}\right) & \phi_{1}^{\prime}\left(l_{22}\right)-\phi_{1}^{\prime}\left(l_{12}\right) \\
\phi_{2}^{\prime}\left(l_{21}\right)-\phi_{2}^{\prime}\left(l_{11}\right) & \phi_{2}^{\prime}\left(l_{22}\right)-\phi_{2}^{\prime}\left(l_{12}\right) \\
\phi_{3}^{\prime}\left(l_{21}\right)-\phi_{3}^{\prime}\left(l_{11}\right) & \phi_{3}^{\prime}\left(l_{22}\right)-\phi_{3}^{\prime}\left(l_{12}\right) \\
\phi_{4}^{\prime}\left(l_{21}\right)-\phi_{4}^{\prime}\left(l_{11}\right) & \phi_{4}^{\prime}\left(l_{22}\right)-\phi_{4}^{\prime}\left(l_{12}\right)
\end{array}\right], \\
{\left[\begin{array}{l}
V_{1} \\
V_{2}
\end{array}\right]=\left(\boldsymbol{\Phi}^{T} \boldsymbol{\Phi}\right)^{-1} \boldsymbol{\Phi}^{T} \tau}
\end{gathered}
$$

Equation (65) is the optimal least squares solution to Eq. 64, assuming all the columns of $\boldsymbol{\Phi}$ are independent. The model can be simplified to the case with one PZT by removing the second column of the Galerkin function dependent matrix in Eq. 64). 


\section{IV.D. Nonlinear Controller in Galerkin Form}

The reference signal $\xi_{r}$ can be approximated using Galerkin functions as $\xi_{r}=\phi^{T}(x) \eta_{r}(t)$ assuming it satisfies the boundary conditions on $\xi$. The control law discussed in Section III.D can be expressed in Galerkin form by minimizing the weighted residual.

$$
\begin{gathered}
s_{\theta}=\dot{\theta}-\dot{\theta_{r}}, \quad s_{\xi}=\phi^{T}\left(\dot{\eta}-\dot{\eta}_{r}\right) \\
m_{12_{g}} \ddot{\eta}_{r}+c_{12_{g}} \dot{\eta}_{r}=K_{\theta}\left(\dot{\theta}-\dot{\theta}_{r}\right) \ell-m_{11_{g}} \ddot{\theta}_{r}-c_{11_{g}} \dot{\theta}_{r} \\
\tau=\int_{0}^{\ell} 2 \phi M_{b}^{\prime \prime} d x=m_{21_{g}} \ddot{\theta}_{r}+m_{22_{g}} \ddot{\eta}_{r}+c_{21_{g}} \dot{\theta}_{r}+2 \delta[E](\eta+\mu \dot{\eta})-K_{\xi} \int_{0}^{\ell} \phi \phi^{T} d x\left(\dot{\eta}-\dot{\eta}_{r}\right)
\end{gathered}
$$

The control effort given in Eq. 67] is used to compute the voltage using Eq. 65).

\section{IV.E. Simulation Results}

In this section, we apply the proposed control law to a cylinder with flexible composite beams as shown in Fig. 4. The model corresponds to the experimental setup described in Section $\mathrm{V}$, whose nominal design parameters are given in Table 1. We simulate numerically the closed-loop system for controlled rotation of the cylinder by applying a voltage to the piezoelectric actuators on the beam. Here we track a sinusoidal signal of amplitude 0.001 radians and frequency $0.02 \mathrm{~Hz}$, and slew from a zero initial attitude to 0.001 radians for different values of $\delta$. We present results for two cases: 1) Configuration 1: with only 'PZT LEFT 1' and 'PZT RIGHT 1' active (see Fig. 44, and 2) Configuration 2: Distributed configuration, with all four PZT actuators active. The numerical implementation of the closed-loop discretized system in ODE form is shown in Fig. 3 .

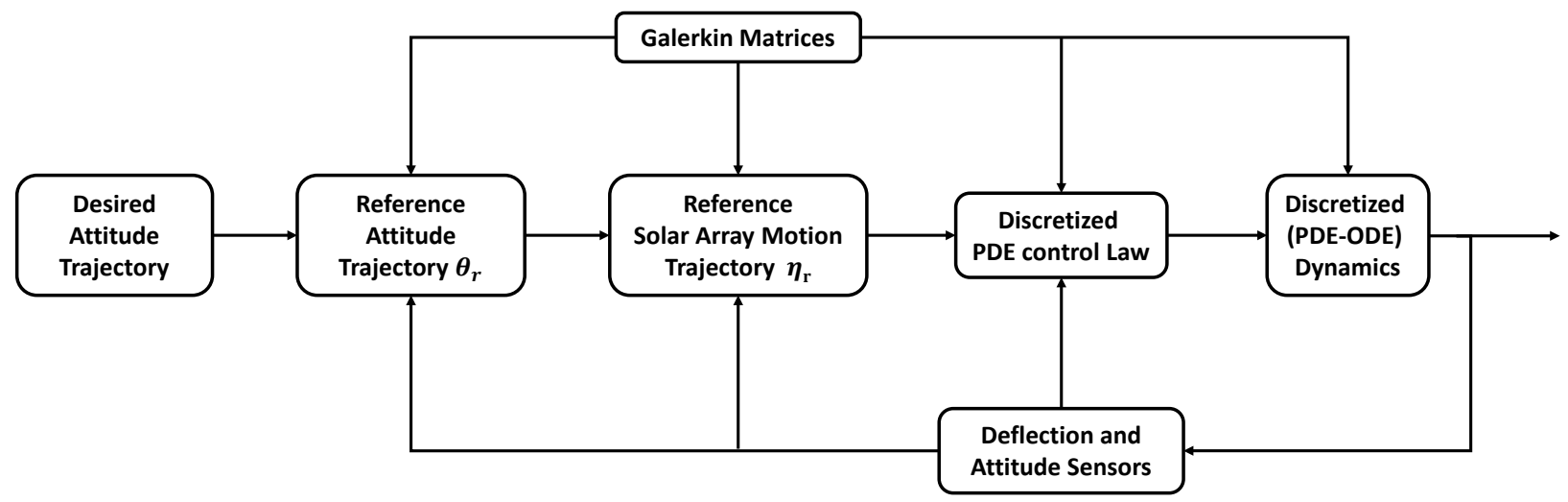

Figure 3: Closed-loop ODE system as implemented in Simulink for simulations.

The Galerkin matrices are computed using Eq. (59) for the configuration shown in Fig. 4. Using the Galerkin matrices, the closed-loop system defined by the ODE given in Eqs. (56)- 596 and Eqs. 66- 67) can be integrated using Euler's method. The Galerkin matrices are computed offline for the nominal design 
Table 1: Physical, geometrical and structural parameters of bus, array, and PZT.

\begin{tabular}{rl|rl|rl}
\hline \hline$\ell$ & $29.7 \times 10^{-2} \mathrm{~m}$ & $E_{b}$ & $68.9 \mathrm{GPa}$ & $E_{p}$ & $66 \mathrm{GPa}$ \\
$t_{b}$ & $0.45 \times 10^{-3} \mathrm{~m}$ & $\rho_{b}$ & $2738 \mathrm{~kg} / \mathrm{m}^{3}$ & $\rho_{p}$ & $7800 \mathrm{~kg} / \mathrm{m}^{3}$ \\
$w$ & $0.036 \mathrm{~m}$ & $\mu$ & $10^{-4}$ & $t_{p}$ & $0.48 \times 10^{-3} \mathrm{~m}$ \\
$d_{31}$ & $190 \times 10^{-12} \mathrm{~m} / \mathrm{V}$ & $\ell_{11}$ & $1.1 \times 10^{-2} \mathrm{~m}$ & $\ell_{21}$ & $8.144 \times 10^{-2} \mathrm{~m}$ \\
$\ell_{12}$ & $8.614 \times 10^{-2} \mathrm{~m}$ & $\ell_{22}$ & $15.858 \times 10^{-2} \mathrm{~m}$ & $\mathrm{r}$ & $0.6 \times 10^{-2}$ \\
$J_{\theta}$ & $1.0759 \times 10^{-6} \mathrm{~m}$ & & & & \\
\hline \hline
\end{tabular}

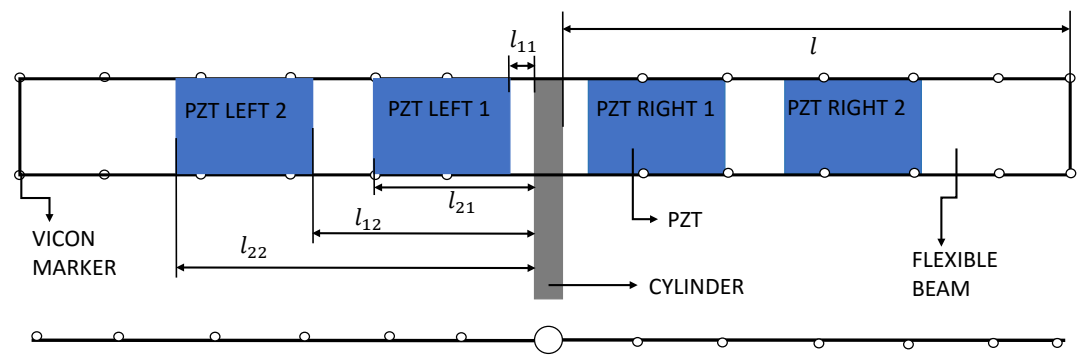

Figure 4: Front and top view of the experimental setup.

parameters. The closed-loop system is simulated using Simulink with the Galerkin matrices. The trajectory tracking and slewing is achieved for $\delta=\{0,0.5,1\}$ with the gain values specified in the figures.

For configuration 1, we present results for $\delta=\{1,0.5,0\}$; see Figs. 57 for trajectory tracking, and Figs. 810 for slewing. If the closed-loop simulation is performed with the same gain values for the three $\delta$ values, it was observed that the tracking error is inversely proportional to the $\delta$ value in the range $[0,1]$. For $\delta=1$, trajectory tracking is achieved exponentially fast. The voltage signals were computed from the control signal offline using Eq. 65. Note that the maximum voltage required to do the tracking and slewing is well within $\pm 200 \mathrm{~V}$, which is the saturation limit of the PZT actuators used in experiments. It is observed that controller 1 predominantly uses the first free vibration mode of the beam to achieve tracking. For slewing results, the time required to reach steady state is increased for smaller $\delta$. Figures 11 , 12 show tracking and slewing in configuration 2. Based on these simulation results, experiments are performed for values of $\delta$ for which control effort is within $\pm 200 \mathrm{~V}$. 


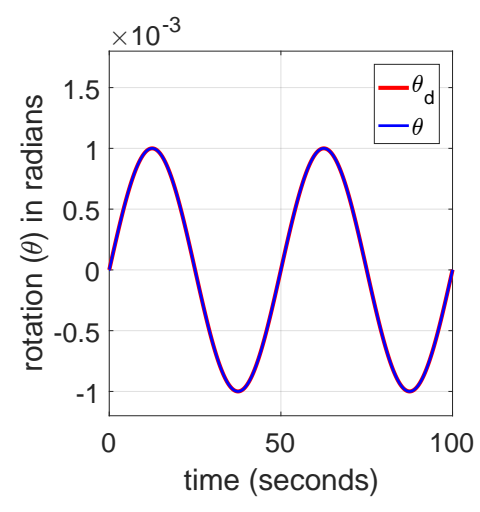

(a) Trajectory tracking

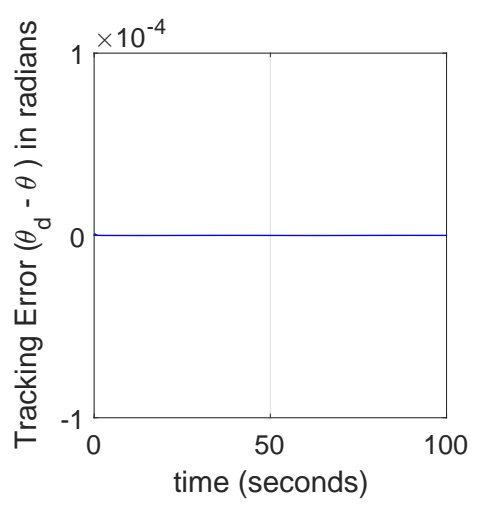

(b) Tracking error

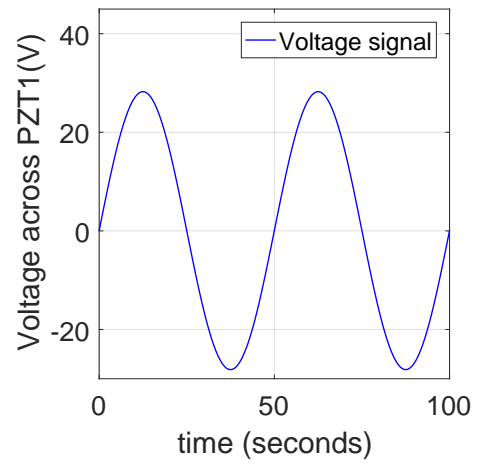

(c) Control effort

Figure 5: Trajectory tracking (simulation) for $\delta=1$ with gains $\lambda_{\theta}=3$, and $K_{\theta}=0.5, K_{\xi}=0.5$.

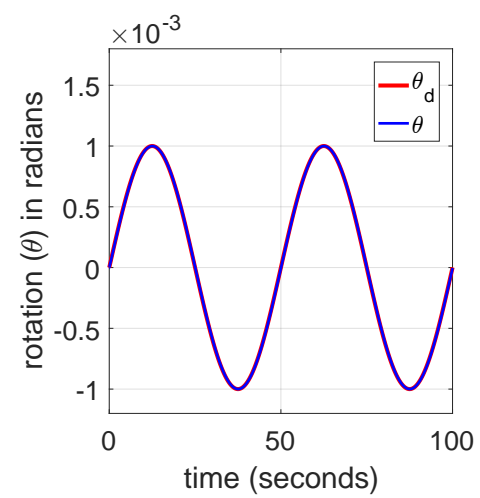

(a) Trajectory tracking

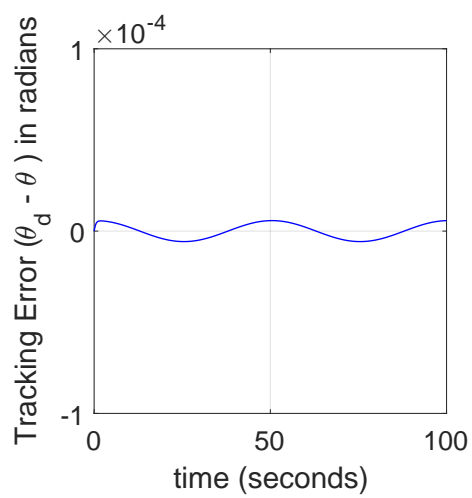

(b) Tracking error

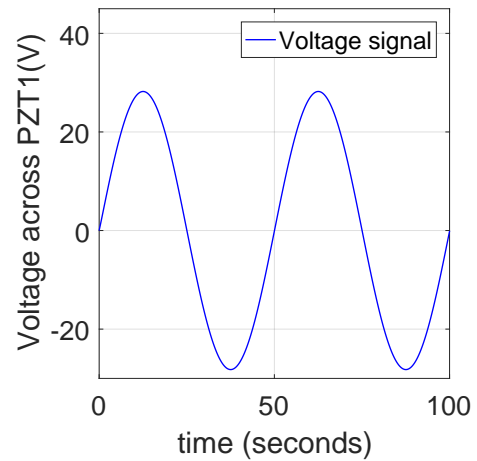

(c) Control effort

Figure 6: Trajectory tracking (simulation) for $\delta=0.5$ with gains $\lambda_{\theta}=3, K_{\theta}=0.5, K_{\xi}=0.5$.

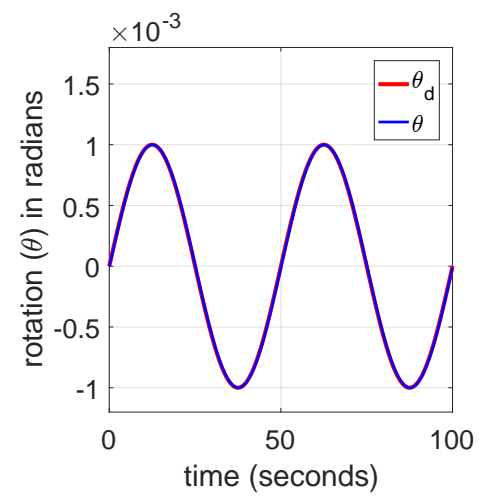

(a) Trajectory tracking

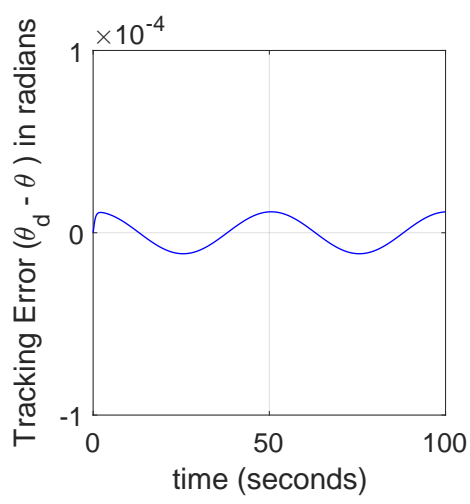

(b) Tracking error

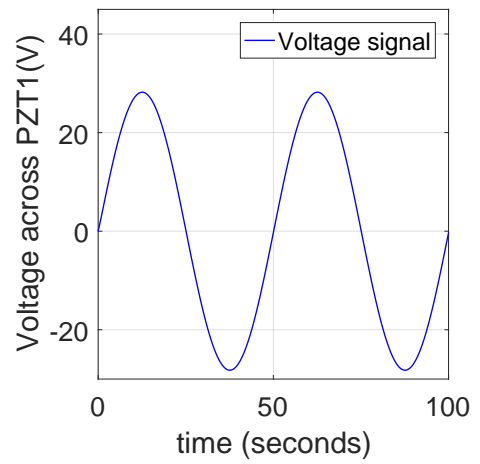

(c) Control effort

Figure 7: Trajectory tracking (simulation) for $\delta=0$ with gains $\lambda_{\theta}=3, K_{\theta}=0.5, K_{\xi}=0.5$. 


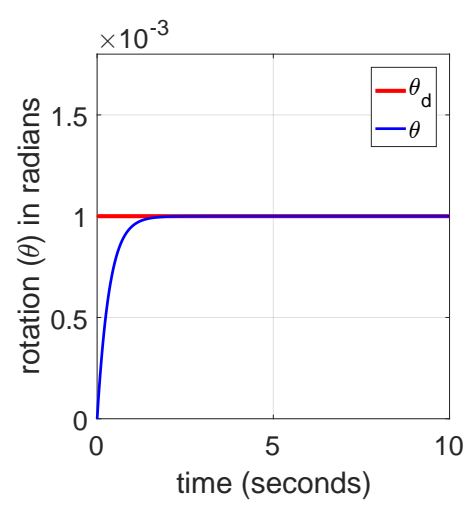

(a) Slewing

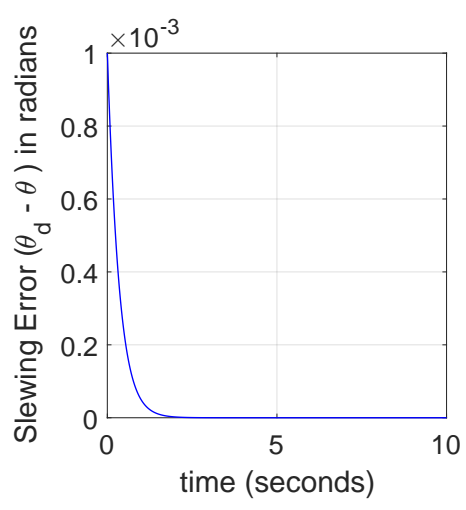

(b) Slewing error

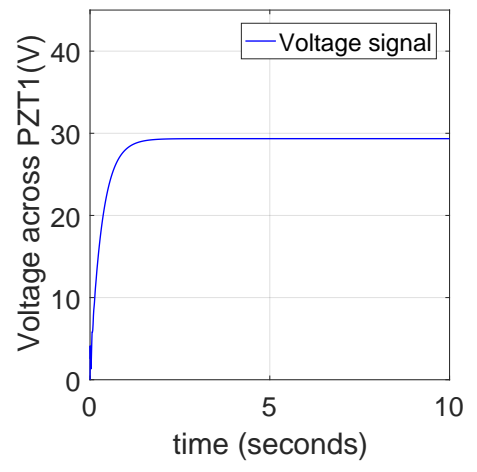

(c) Control effort

Figure 8: Slewing (simulation) for $\delta=1$ with gains $\lambda_{\theta}=3, K_{\theta}=0.5, K_{\xi}=0.5$.

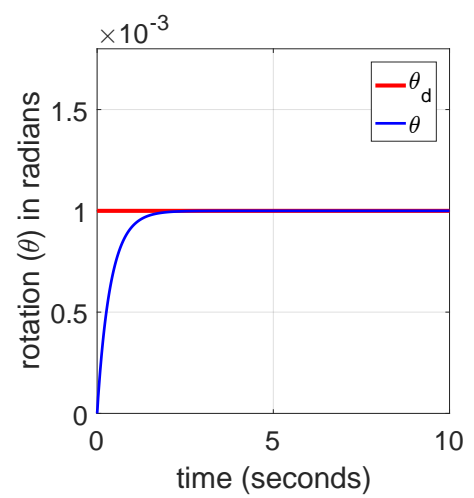

(a) Slewing

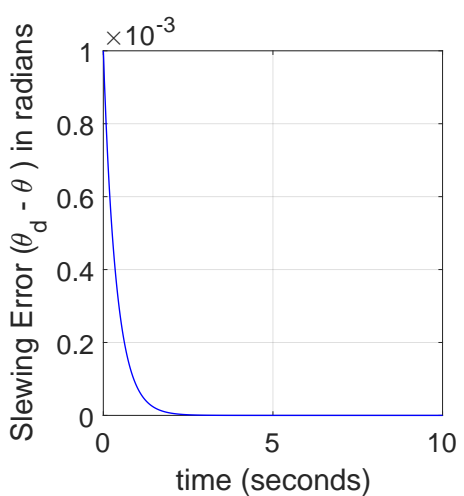

(b) Slewing error

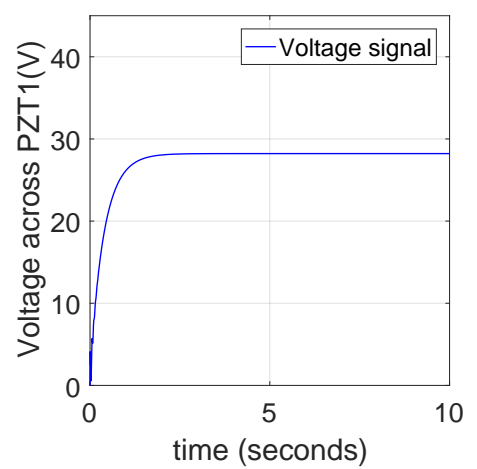

(c) Control effort

Figure 9: Slewing (simulation) for $\delta=0.5$ with gains $\lambda_{\theta}=3, K_{\theta}=0.5, K_{\xi}=0.5$.

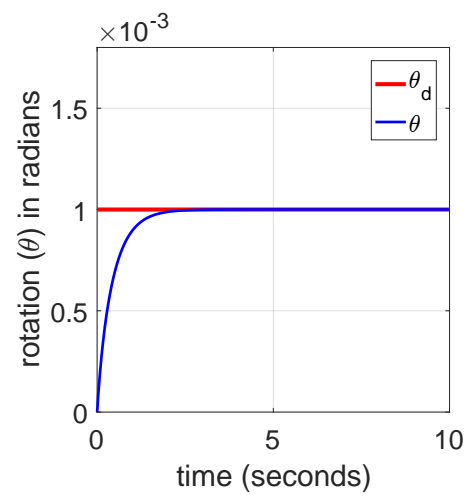

(a) Slewing

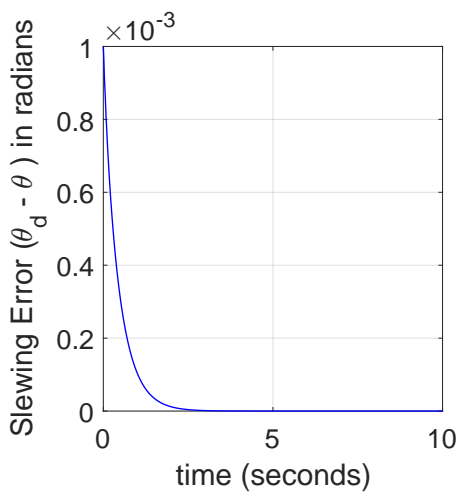

(b) Slewing error

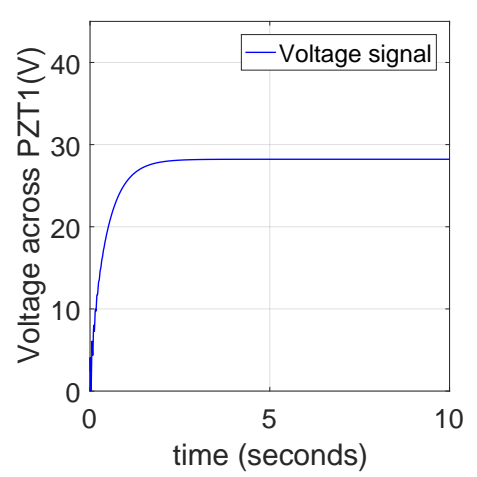

(c) Control effort

Figure 10: Slewing (simulation) for $\delta=0$ with gains $\lambda_{\theta}=3, K_{\theta}=0.5, K_{\xi}=0.5$. 


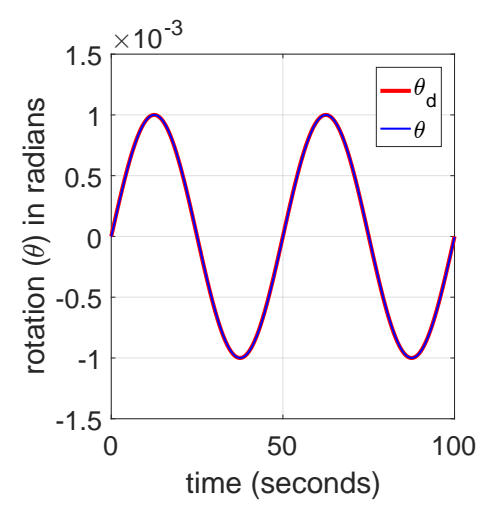

(a) Trajectory tracking

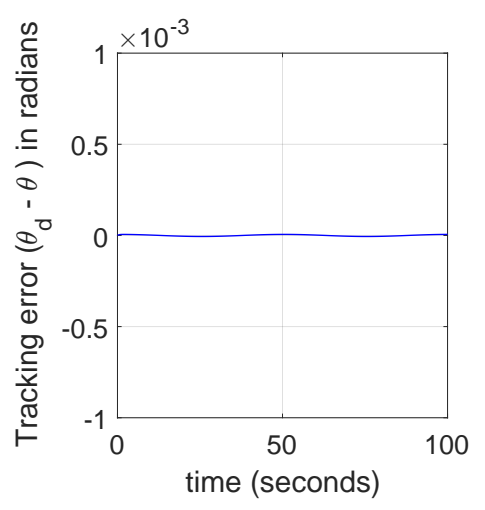

(b) Tracking error

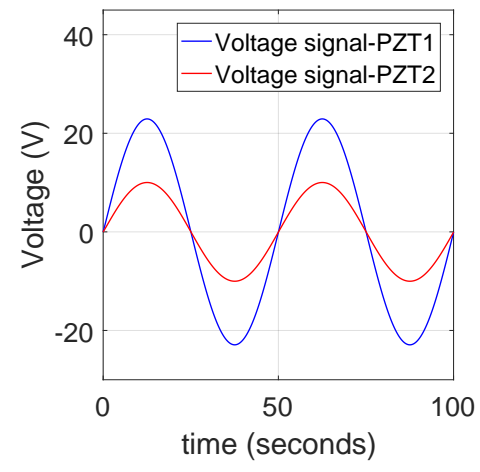

(c) Control effort

Figure 11: Trajectory tracking (simulation) for $\delta=0.5$ in configuration 2 with gains $\lambda_{\theta}=3, K_{\theta}=0.5$, $K_{\xi}=0.5$.

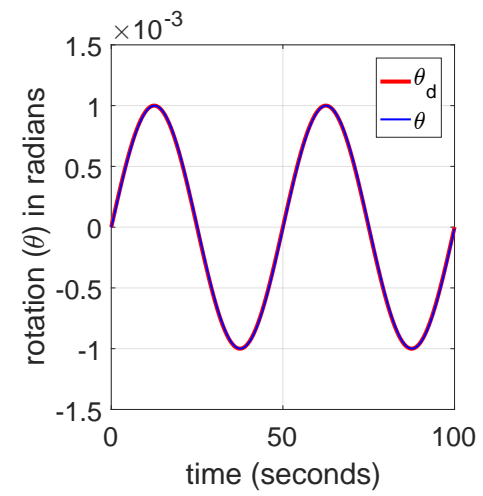

(a) Trajectory tracking

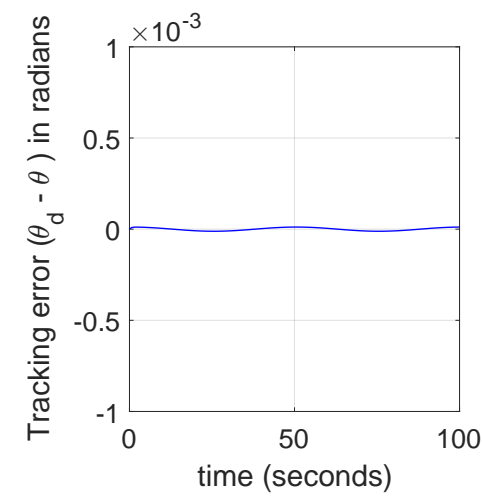

(b) Tracking error

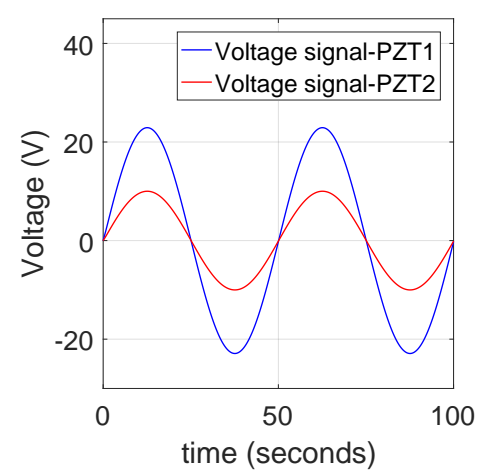

(c) Control effort

Figure 12: Trajectory tracking (simulation) for $\delta=0$ in configuration 2 with gains $\lambda_{\theta}=3, K_{\theta}=0.5$, $K_{\xi}=0.5$.

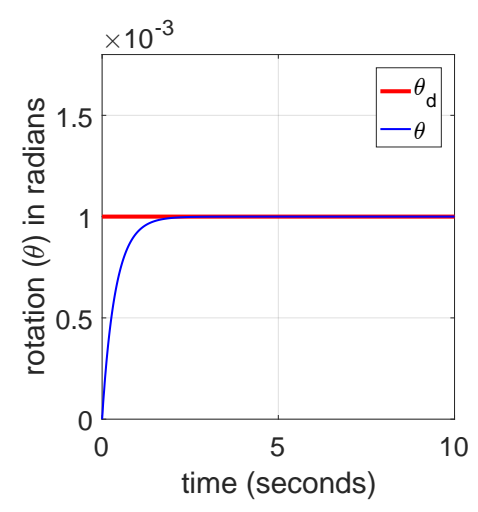

(a) Slewing

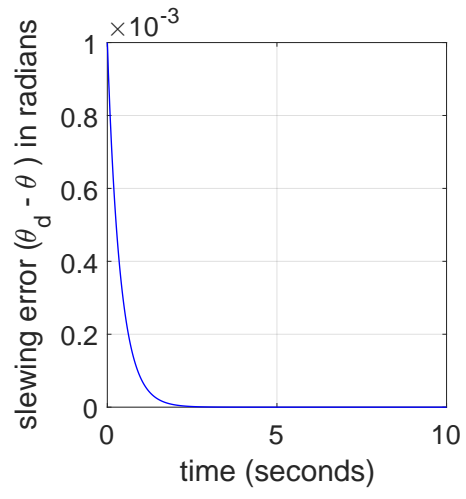

(b) Slewing error

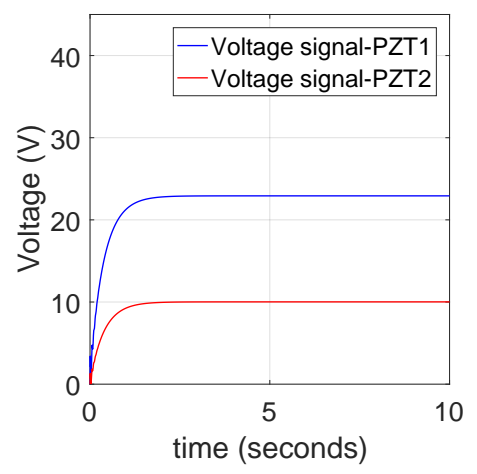

(c) Control effort

Figure 13: Slewing (simulation) for $\delta=0.5$ in configuration 2 with gains $\lambda_{\theta}=3, K_{\theta}=0.5, K_{\xi}=0.5$. 


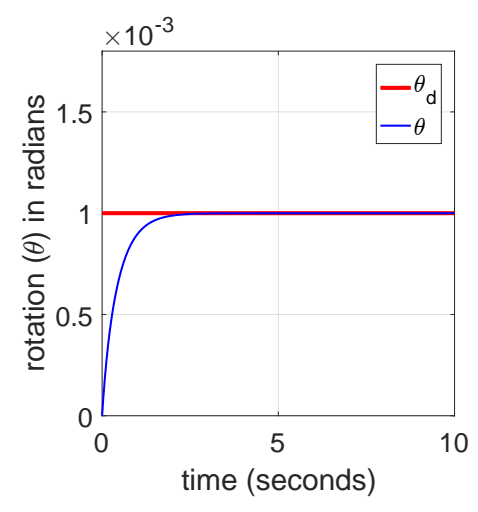

(a) Slewing

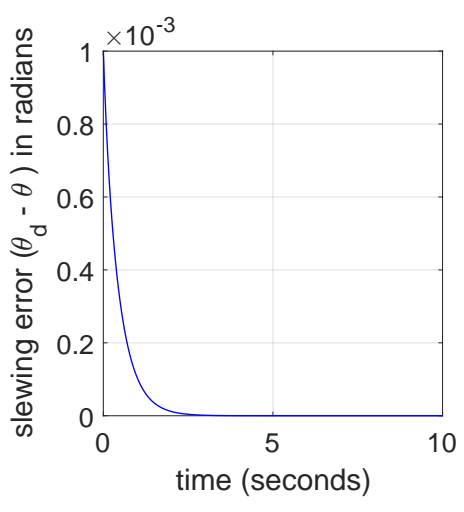

(b) Slewing error

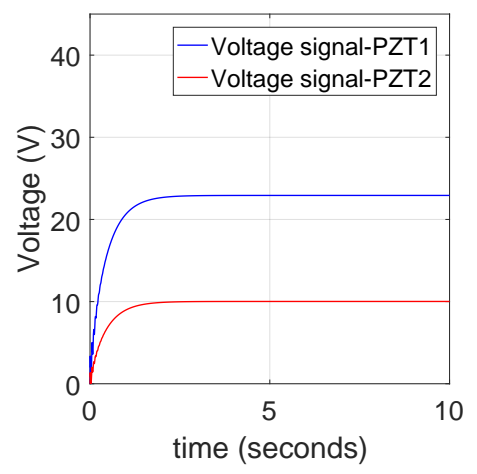

(c) Control effort

Figure 14: Slewing (simulation) for $\delta=0$ in configuration 2 with hains $\lambda_{\theta}=3, K_{\theta}=0.5, K_{\xi}=0.5$.

\section{Experiments}

\section{V.A. Actuator Model Validation}

The quasi-static actuator model in Eq. (11) is validated using a cantilever beam setup (see Fig. 15. The system parameters of the setup are given are $\ell_{b a}=24.4 \times 10^{-2} \mathrm{~m}, \ell_{1 p a}=0.9 \times 10^{-2} \mathrm{~m}, \ell_{p a}=7.244 \times 10^{-2}$ m. The beam and PZT density are listed in Table 1

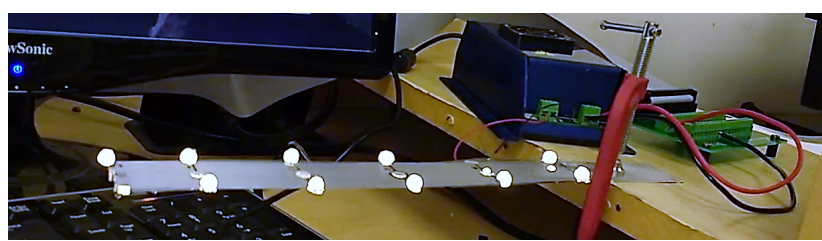

(a) Beam test setup

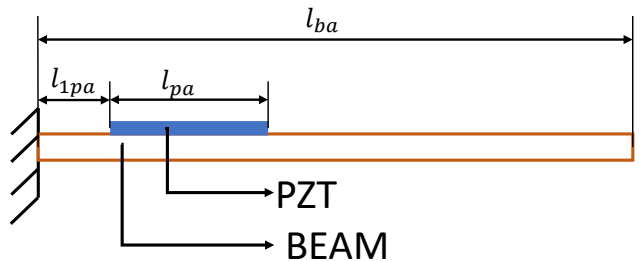

(b) Beam schematic

Figure 15: Open-loop beam experimental setup.

The cantilever beam dynamics in PDE form is given in Eq. (68) (see [12]). The Galerkin method was used to discretize the PDE equation to form the ODE in Eq. 69.)

$$
\begin{array}{r}
m_{R} \ddot{\xi}+\left(E I \xi^{\prime \prime}+\mu E I \dot{\xi}^{\prime \prime}\right)^{\prime \prime}-M_{b}^{\prime \prime}(x, t)=0 \\
\left(\int_{0}^{\ell_{a b}} m_{R} \phi \phi^{T} d x\right) \ddot{\eta}+\left(\int_{0}^{\ell_{a b}} \phi\left(E I \phi^{\prime \prime T}\right)^{\prime \prime} d x\right)(\eta+\mu \dot{\eta})-\int_{0}^{\ell_{a b}} \phi M_{b}^{\prime \prime}(x, t) d x=0
\end{array}
$$

In Figs. 16, we compare the open-loop response of the beam computed from simulations against results measured using the Vicon motion capture system during experiments at the tip of the beam. 


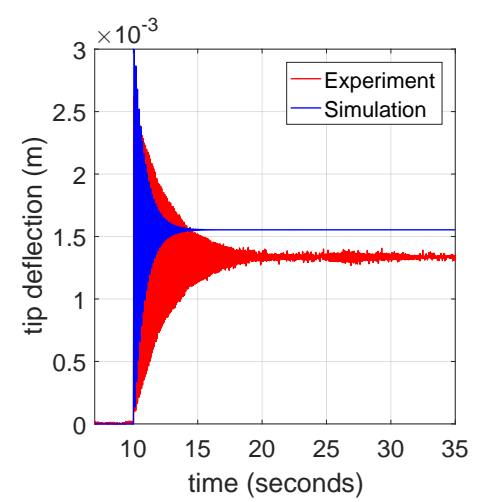

(a) Response to step input

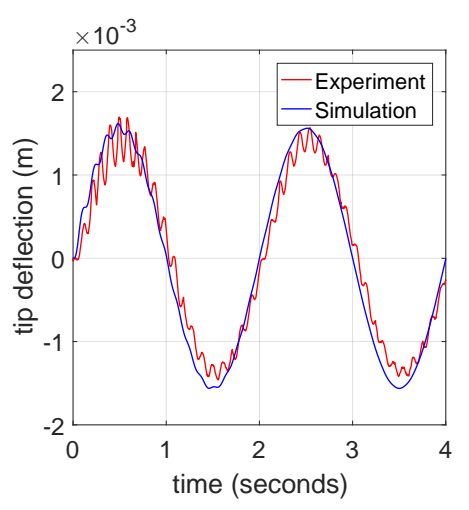

(b) Response to a sinusoidal input

Figure 16: Open-loop experiment vs simulation, beam tip deflection.

For a sinusoidal input, the frequency of the simulated response matches experimental results. The amplitude of the response is not an exact match because 1) we assume there is perfect bonding between the beam and PZT, 2) the beam is fixed at the root using a 'C-clamp', which is not an ideal cantilever beam, and 3) the Vicon marker effect on the beam is not modeled. We proceed and use this model in the real time experiments as the difference between the simulation and experiments can be compensated with additional control effort (it can be considered as a bounded uncertainty at the input of the system Eq. (25)).

\section{V.B. SASA Experimental Setup}

The experimental setup is shown in Fig. 18a, which includes SASA setup, Vicon system, amplifiers, and the controller. The top and side view of the SASA setup are shown in Fig. 18. The setup has a cylinder, a beam, and two plates. The properties of the beam and PZT used are given in Table 1 The cylinder has a slot machined using wire cut Electrical Discharge Machining (EDM), through which beam is fitted to the cylinder. The ball bearings are secured to the plates using an interference fit and the cylinder is press fit into the inner bearing race. Two ball bearings are used to resist torques perpendicular to the axis of the cylinder, and to allow rotation about the cylinder axis. Here we used a T120-A4E-602 PZT sheet manufactured by Piezo Systems, Inc. with a maximum input voltage of $\pm 200 \mathrm{~V}$.

Two PZTs are bonded on each side of the beam using superglue ${ }^{\mathrm{a}}$ (the above cantilever beam experiments used this bonding agent as well), for asymmetric actuation. A voltage signal is supplied to the PZT amplifiers using a Humusoft MF624 controller, which can produce up to $\pm 10 \mathrm{~V}$. We use linear amplifiers manufactured by Piezo systems to amplify the voltage supplied by the controller before applying it across the PZTs. The real-time code for experiment was setup in Matlab using Simulink ${ }^{\circledR}$ Desktop real-time ${ }^{\text {TM }}$ toolbox. For

\footnotetext{
${ }^{\mathrm{a}}$ Loctite ${ }^{\circledR}$ Super Glue
} 
computation of derivatives and integrals we used the discrete time function blocks ${ }^{\mathrm{b}}$ available in Simulink ${ }^{\circledR}$. The flowchart in Fig. 20 shows the flow of the real-time implementation. As seen in Fig. 20, we use three Vicon cameras to measure the beam deflection and cylinder rotation. The Vicon system communicates with the controller (i.e., a desktop computer with the Humusoft controller) using a local wireless area network.

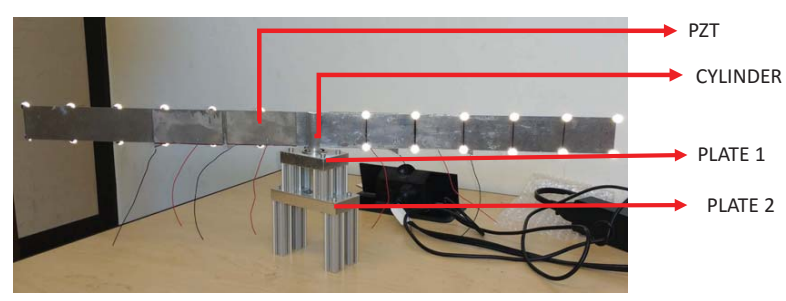

(a) Side view

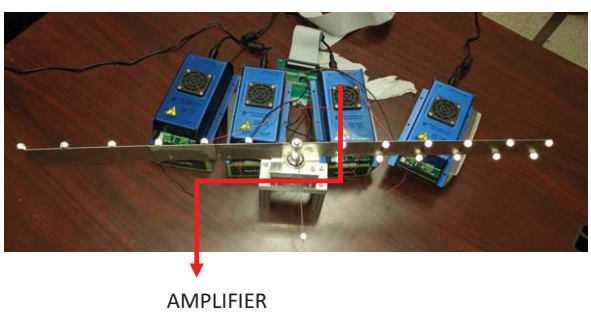

(b) Top view with amplifiers

Figure 17: SASA setup views

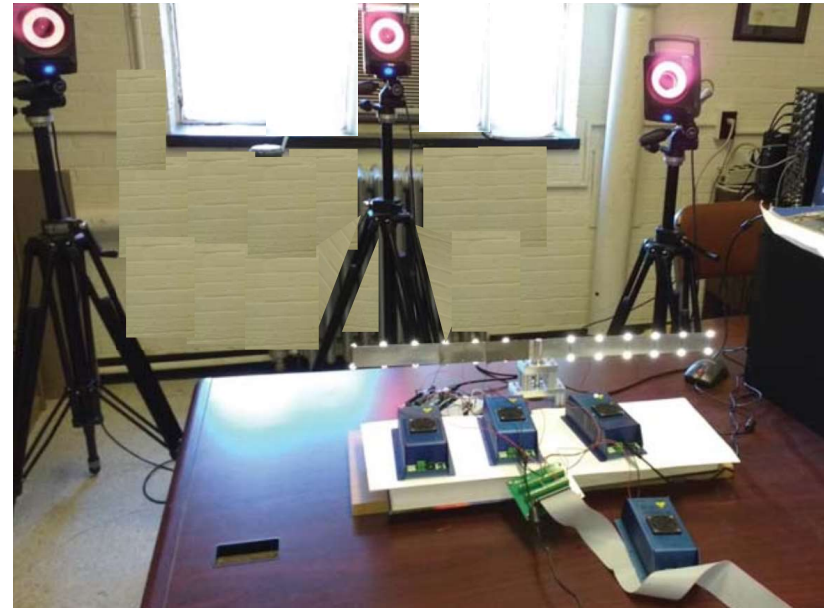

(a) SASA experimental setup

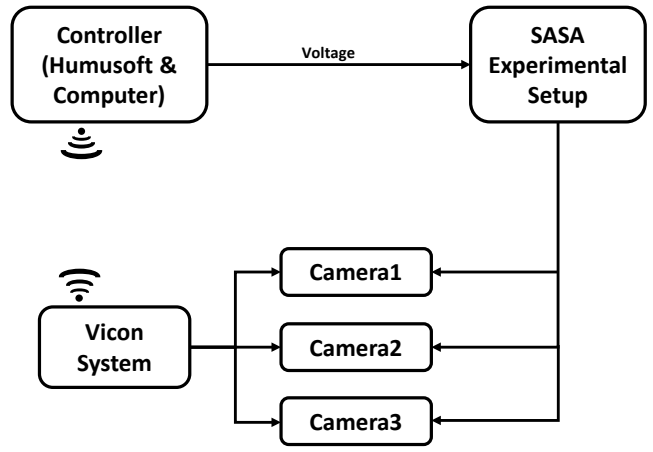

(b) Schematic of SASA test setup

Figure 18: SASA setup

The properties in Table 1 are estimated using physical properties of the aluminum beam, cylinder, and steel ball bearings. The experimental system is built to be symmetric so that measurements from a single beam can be used to compute control effort during the real-time experiments. To use the dynamics and control law derived, we transform the Vicon system world coordinate system, $e_{a_{1}}$ and $e_{a_{2}}$, to an inertial coordinate system fixed to the center of the cylinder, $e_{c_{1}}$ and $e_{c_{2}}$. Note that in Figs. 4 18a, and 19, there are 12 Vicon markers at 6 locations on each beam. The $x$ and $y$ coordinates of these Vicon markers are measured in $e_{a_{1}}$ and $e_{a_{2}}$, and then transformed to coordinate system $e_{c_{1}}$ and $e_{c_{2}}$ to compute deflection at these locations. The deflection at the 6 Vicon marker locations is computed by solving an inverse kinematic problem given in Eq. (70), where $\theta$ is measured using the Vicon marker as shown in Fig. 19

\footnotetext{
bhttp://www.mathworks.com/help/simulink/discrete.html
} 


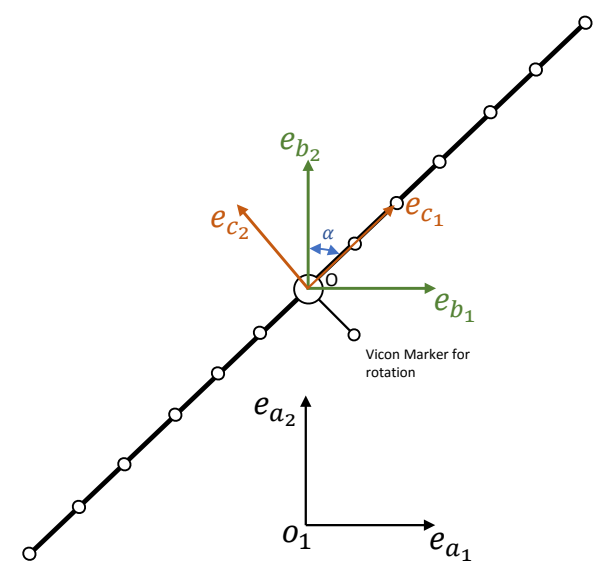

Figure 19: Top view of the setup showing coordinate systems used in experiments.

$$
\left[\begin{array}{c}
r+x \\
\xi
\end{array}\right]=\left[\begin{array}{cc}
\cos (\theta) & \sin (\theta) \\
-\sin (\theta) & \cos (\theta)
\end{array}\right]\left[\begin{array}{l}
x \\
y
\end{array}\right]
$$

Let the deflection measured at the 6 Vicon marker locations (with distances from root $\left[\ell_{v_{1}} \ell_{v_{2}} \ell_{v_{3}} \ell_{v_{4}} \ell_{v_{5}} \ell_{v_{6}}\right]$ ) using the Vicon system be $\xi_{\text {exp }}=\left[\begin{array}{llllll}\xi_{1} & \xi_{2} & \xi_{3} & \xi_{4} & \xi_{5} & \xi_{6}\end{array}\right]$. The measurements $\xi_{\text {exp }}$ are used to compute $\eta$ for the four Galerkin functions in Eq. 171). The derivatives $\dot{\theta}$ and $\dot{\eta}$ are estimated using discrete derivatives of the computed $\theta$ and $\eta$ values from Vicon measurements.

$$
\xi_{\exp }^{T}=\left[\begin{array}{llll}
\phi_{1}\left(\ell_{v_{1}}\right) & \phi_{2}\left(\ell_{v_{1}}\right) & \phi_{3}\left(\ell_{v_{1}}\right) & \phi_{4}\left(\ell_{v_{1}}\right) \\
\phi_{1}\left(\ell_{v_{2}}\right) & \phi_{2}\left(\ell_{v_{2}}\right) & \phi_{3}\left(\ell_{v_{2}}\right) & \phi_{4}\left(\ell_{v_{2}}\right) \\
\phi_{1}\left(\ell_{v_{3}}\right) & \phi_{2}\left(\ell_{v_{3}}\right) & \phi_{3}\left(\ell_{v_{3}}\right) & \phi_{4}\left(\ell_{v_{3}}\right) \\
\phi_{1}\left(\ell_{v_{4}}\right) & \phi_{2}\left(\ell_{v_{4}}\right) & \phi_{3}\left(\ell_{v_{4}}\right) & \phi_{4}\left(\ell_{v_{4}}\right) \\
\phi_{1}\left(\ell_{v_{5}}\right) & \phi_{2}\left(\ell_{v_{5}}\right) & \phi_{3}\left(\ell_{v_{5}}\right) & \phi_{4}\left(\ell_{v_{5}}\right) \\
\phi_{1}\left(\ell_{v_{6}}\right) & \phi_{2}\left(\ell_{v_{6}}\right) & \phi_{3}\left(\ell_{v_{6}}\right) & \phi_{4}\left(\ell_{v_{6}}\right)
\end{array}\right] \eta
$$

\section{V.C. SASA Open-loop Simulation versus Experiments}

For the open-loop results, we measure the bus rotation due to an applied voltage across 'PZT RIGHT 1' and 'PZT LEFT 1' on the beam. The estimation errors are seen clearly in Fig. 21, which illustrates a comparison of open-loop simulation and experimental results. The response to a sinusoidal input is a good match. For the step input, simulations predict larger rotation. This difference is due to the estimation of system parameters and not modeling the ball bearing friction. In spite of the modeling errors, the closed-loop system achieves the control objectives (see Section. V.D for details). 


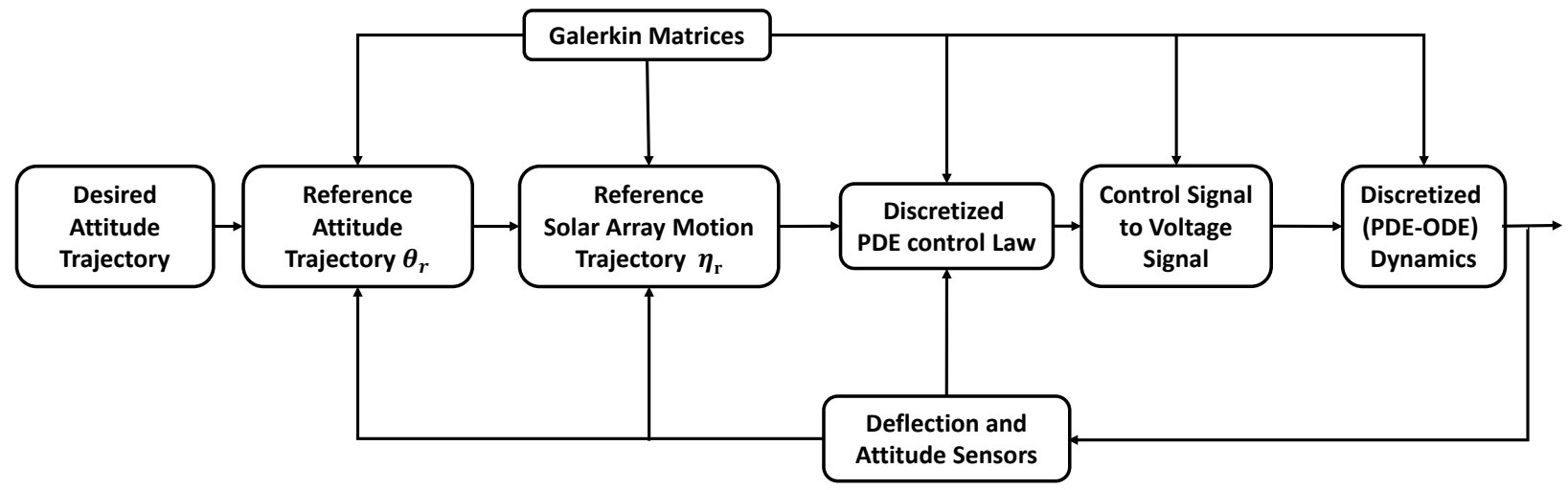

Figure 20: Closed-loop ODE system as implemented in Simulink for experiments.

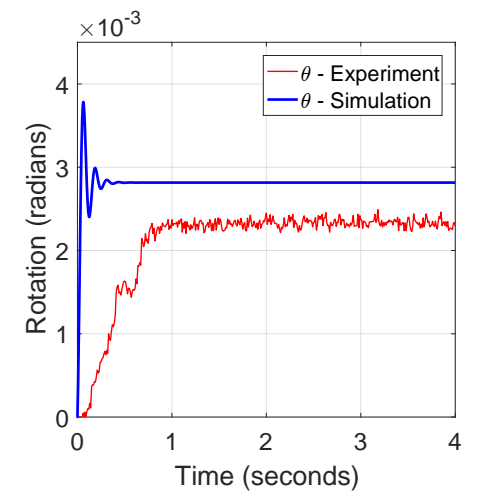

(a) Step response for an input of $100 \mathrm{~V}$

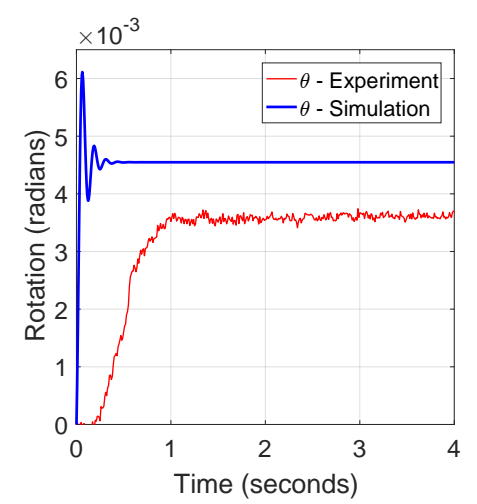

(b) Step response for an input of $160 \mathrm{~V}$

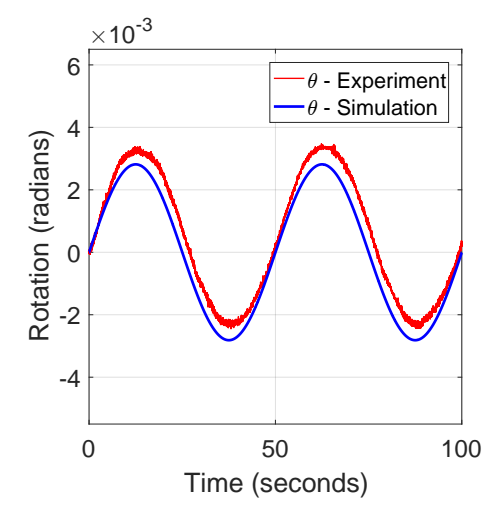

(c) Sinusoidal response for an input of amplitude $100 \mathrm{~V}$.

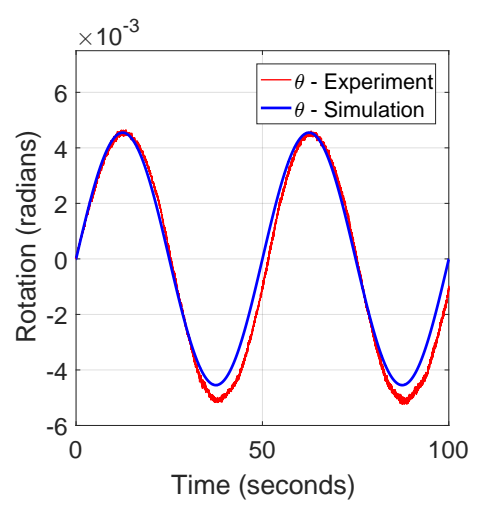

(d) Sinusoidal response for an input of amplitude $160 \mathrm{~V}$

Figure 21: Open-loop experiment versus simulation, bus rotation.

\section{V.D. Control Experiment Results}

The experimental setup described above is used to test the control law. Based on the simulation results, we test the controller in configuration 1 for $\delta=\{1,0.5,0\}$ (Figs. 22 29) and in configuration 2 for $\delta=\{0.5,0\}$ (Figs. 28 31. Similar to the simulation, we track a sinusoidal signal of amplitude 0.001 radians, with a 
frequency of $0.02 \mathrm{~Hz}$, and slew to 0.001 radians from a zero initial angle. As mentioned earlier, the realtime code was implemented using Simulink Desktop real-time toolbox. For all the $\delta$ values listed earlier we achieve the control objectives. The measurements are made and control signal is applied to PZTs at the same frequency $(100 \mathrm{~Hz})$ during the experiment. For the trajectory being tracked, frequencies around $50 \mathrm{HZ}$ to $60 \mathrm{~Hz}$ were exciting the a natural mode of the entire setup. In configuration 2, the PZTs on each side become more active as the $\delta$ value is reduced toward zero. Note that the controllers work notwithstanding the modeling errors and parametric uncertainties. The control effort computed by the control algorithm during experiments is at least 20 Volts more than predicted during simulations.

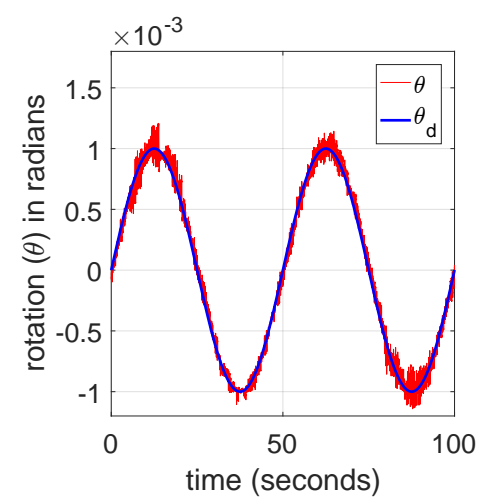

(a) Trajectory tracking

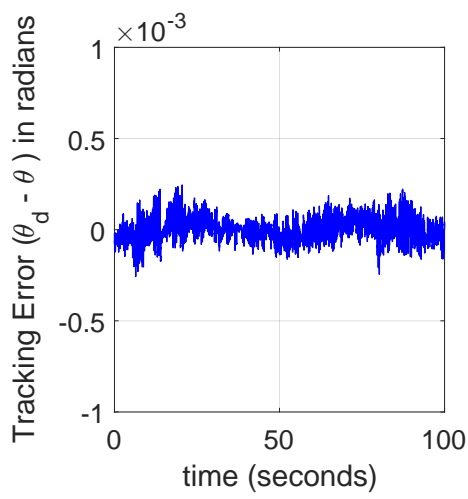

(b) Tracking error

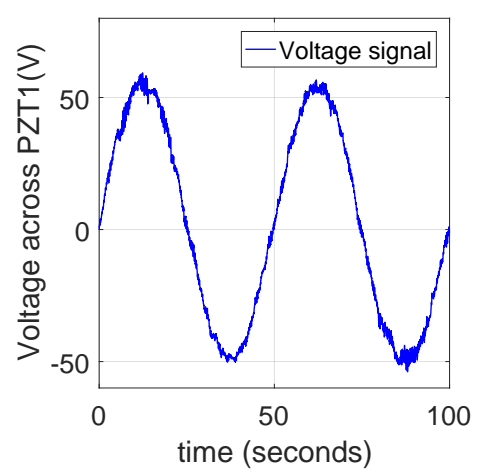

(c) Control effort

Figure 22: Trajectory tracking (experiment) for $\delta=1$ with gains $\lambda_{\theta}=1.5, K_{\theta}=0.5, K_{\xi}=0.5$.

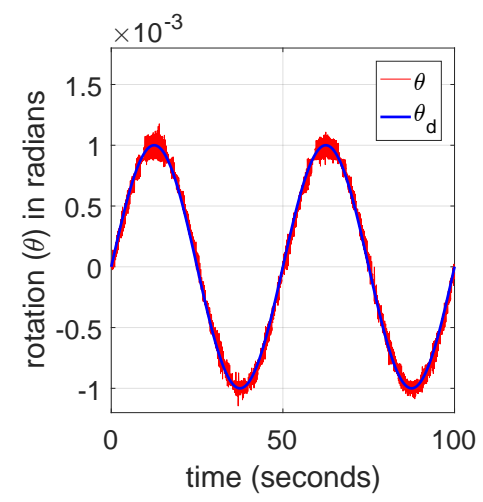

(a) Trajectory tracking

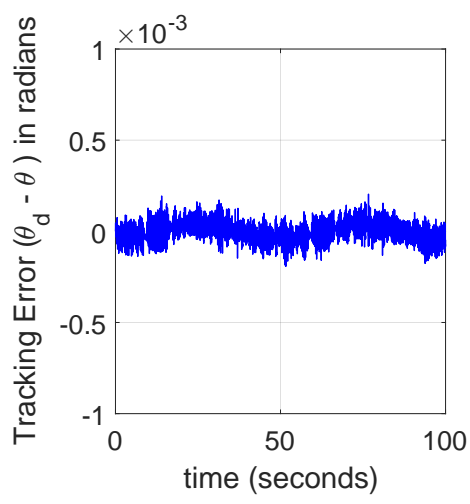

(b) Tracking error

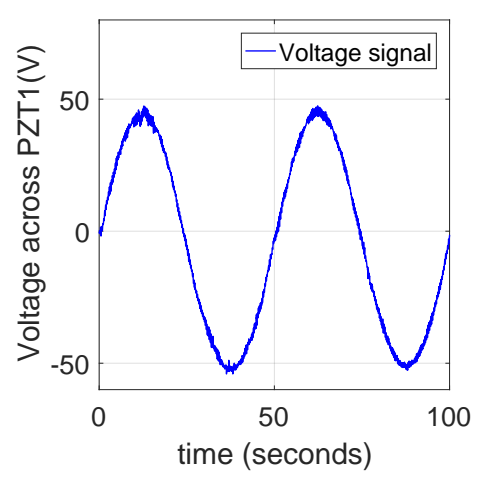

(c) Control effort

Figure 23: Trajectory tracking (experiment) for $\delta=0.5$ with gains $\lambda_{\theta}=1.5, K_{\theta}=0.5, K_{\xi}=0.5$. 


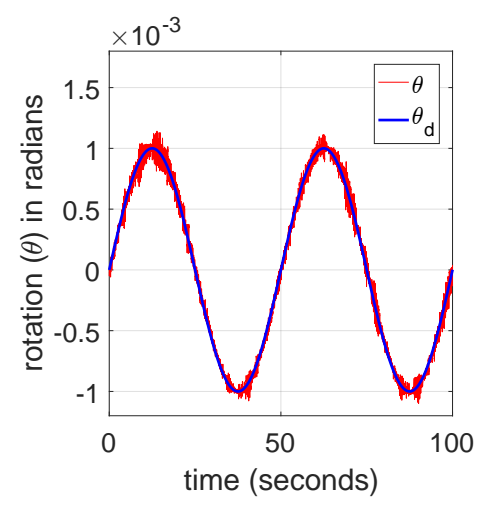

(a) Trajectory tracking

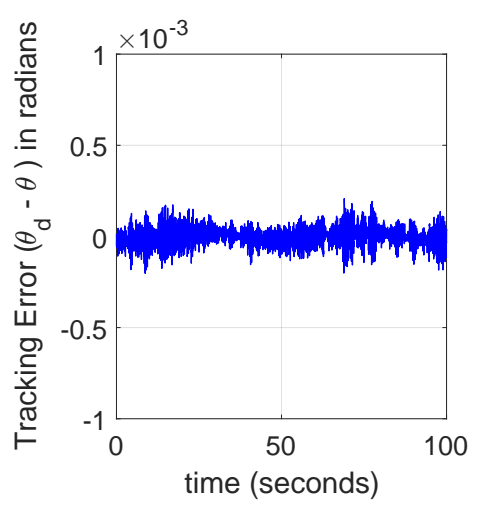

(b) Tracking error

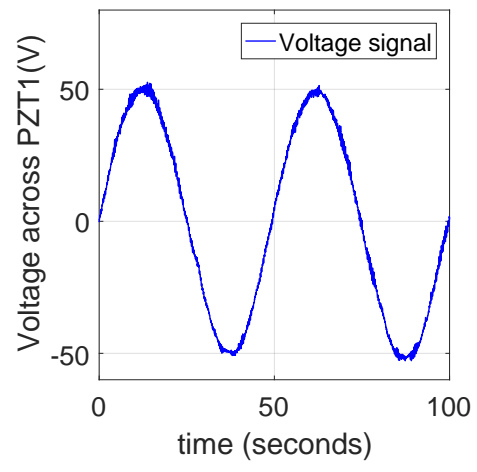

(c) Control effort

Figure 24: Trajectory tracking (experiment) for $\delta=0$ with gains $\lambda_{\theta}=2, K_{\theta}=0.5, K_{\xi}=0.5$.

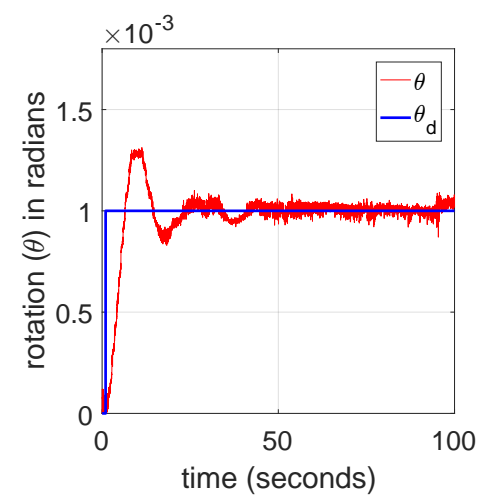

(a) Slewing

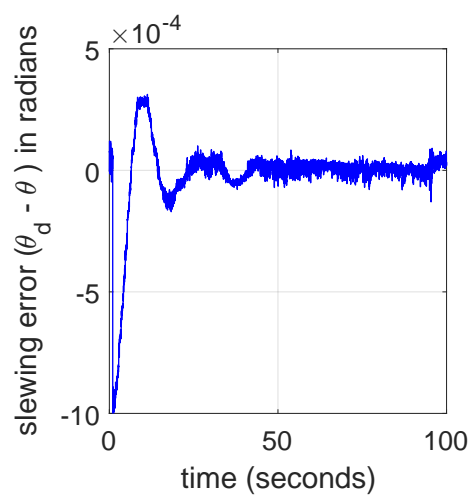

(b) Slewing error

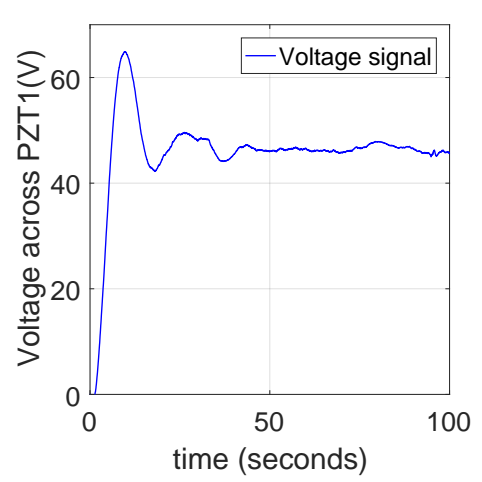

(c) Control effort

Figure 25: Slewing (experiment) for $\delta=1$ with gains $\lambda_{\theta}=1, K_{\theta}=0.25, K_{\xi}=0.5$.

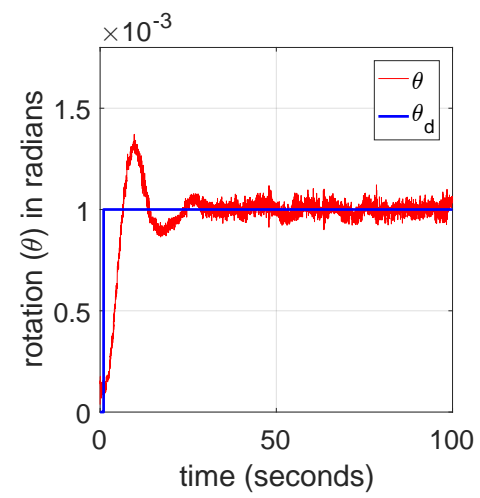

(a) Slewing

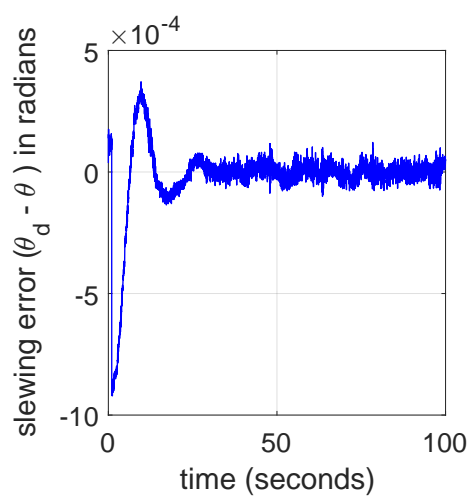

(b) Slewing error

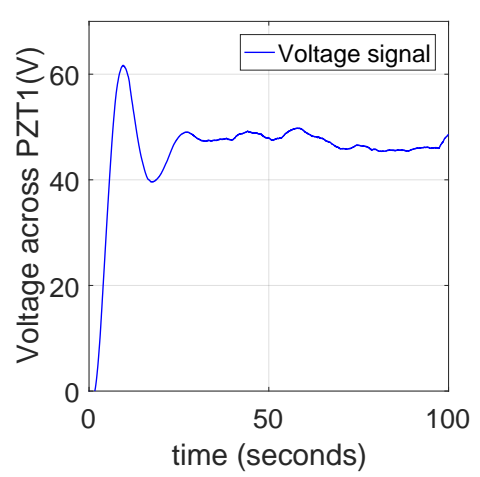

(c) Control effort

Figure 26: Slewing (experiment) for $\delta=0.5$ with gains $\lambda_{\theta}=1, K_{\theta}=0.25, K_{\xi}=0.5$. 


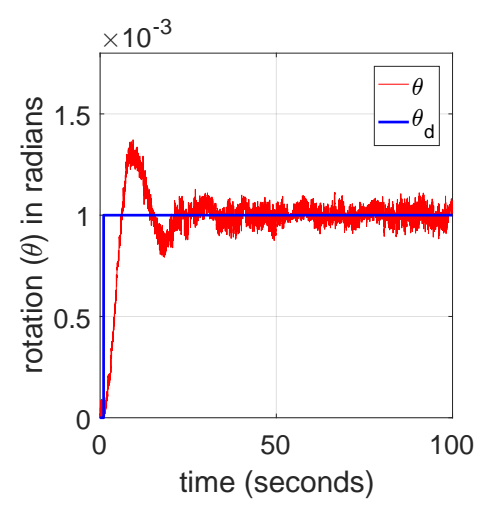

(a) Slewing

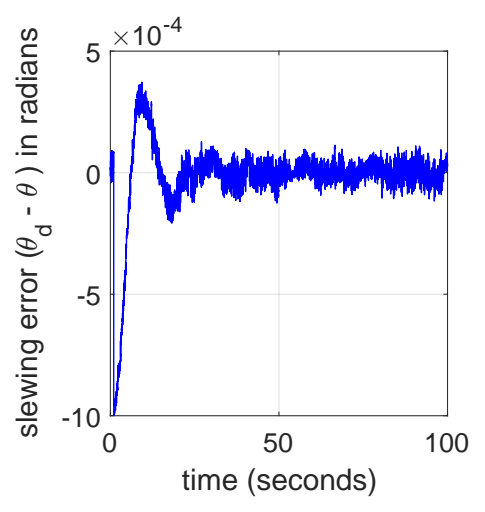

(b) Slewing error

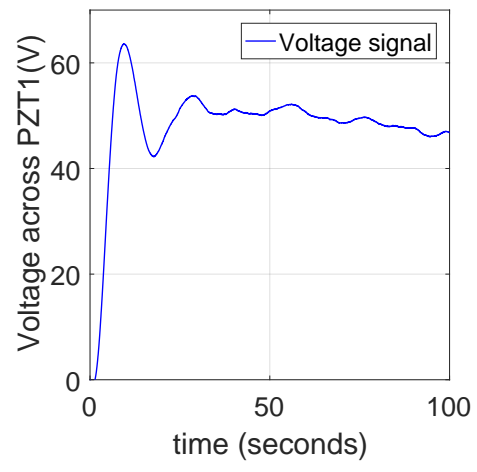

(c) Control effort

Figure 27: Slewing (experiment) for $\delta=0$ with gains $\lambda_{\theta}=1, K_{\theta}=0.25, K_{\xi}=0.5$.

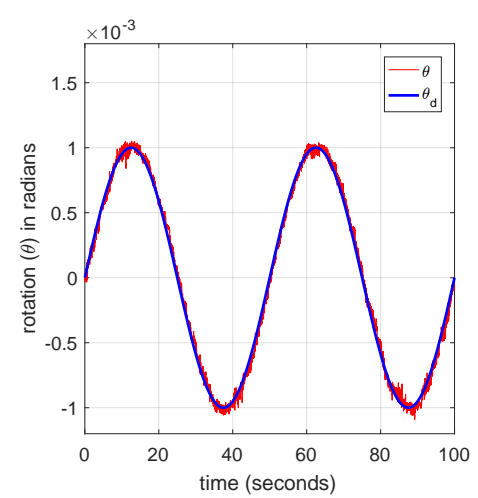

(a) Trajectory tracking

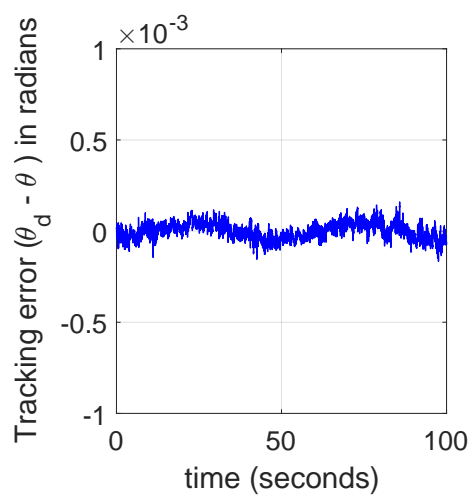

(b) Tracking error

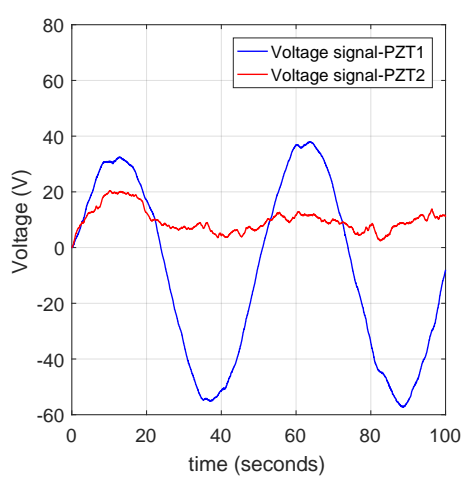

(c) Control effort

Figure 28: Trajectory tracking (experiment) for $\delta=0.5$ in configuration 2 with gains $\lambda_{\theta}=2, K_{\theta}=0.5$, $K_{\xi}=0.5$.

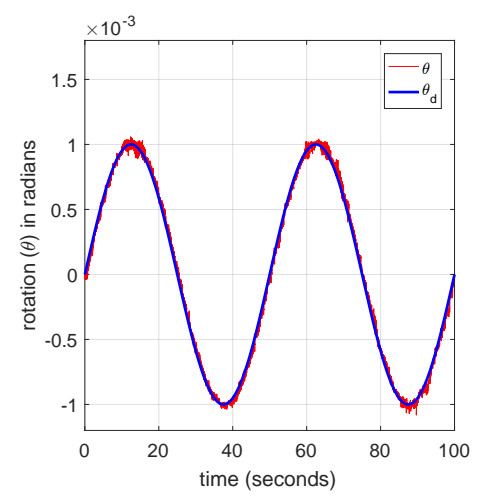

(a) Trajectory tracking

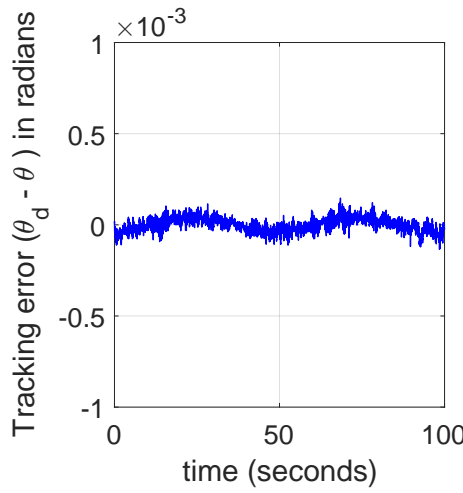

(b) Tracking error

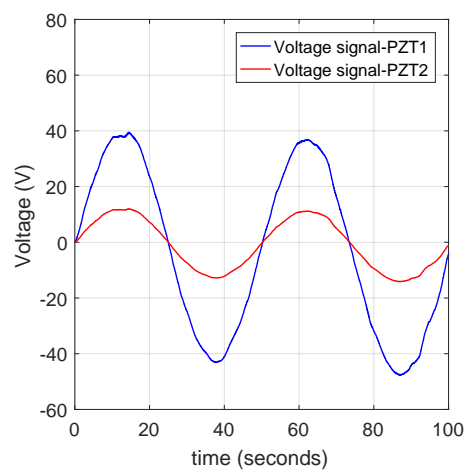

(c) Control effort

Figure 29: Trajectory tracking (experiment) for $\delta=0$ in configuration 2 with gains $\lambda_{\theta}=2, K_{\theta}=0.5$, $K_{\xi}=0.5$. 


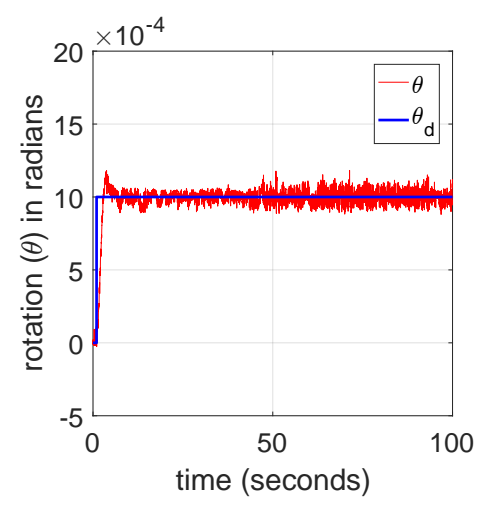

(a) Slewing

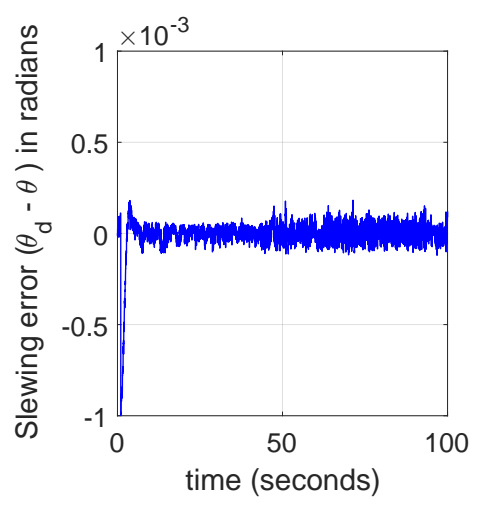

(b) Slewing error

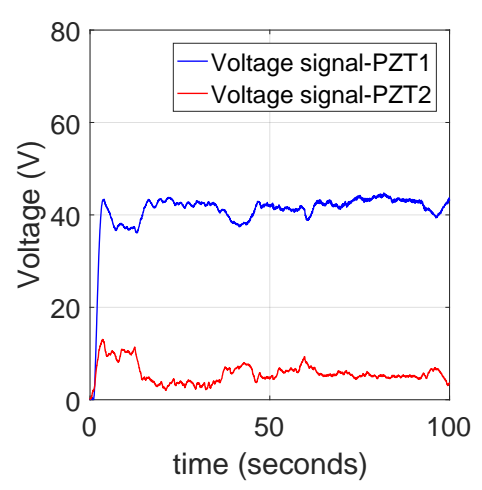

(c) Control effort

Figure 30: Slewing (experiment) for $\delta=0.5$ in configuration 2 with gains $\lambda_{\theta}=2, K_{\theta}=0.5, K_{\xi}=0.5$.

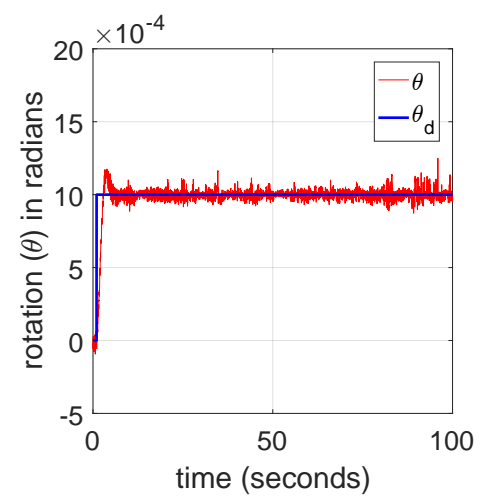

(a) Slewing

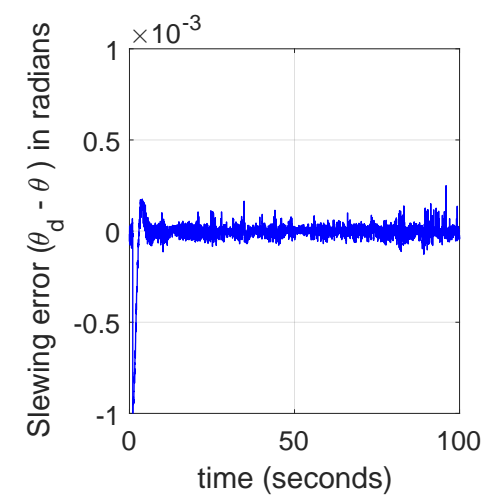

(b) Slewing error

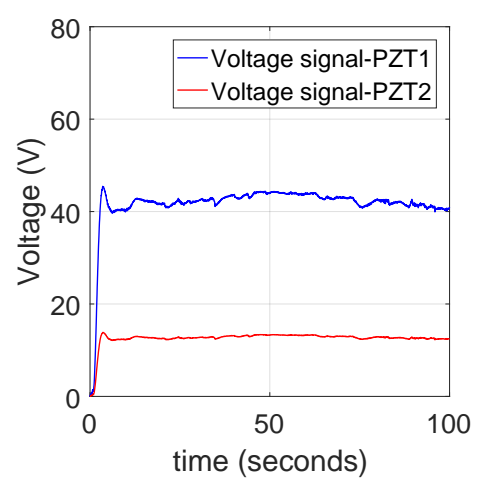

(c) Control effort

Figure 31: Slewing (experiment) for $\delta=0$ in configuration 2 with gains $\lambda_{\theta}=2, K_{\theta}=0.5, K_{\xi}=0.5$.

\section{Conclusion}

In this paper, we derived nonlinear equations of motion that describe the one-degree-of-freedom rotation of a spacecraft with Strain-Actuated Solar Arrays (SASA), and presented a novel control technology with a new Ordinary Differential Equations (ODE)-Partial Differential Equation model (PDE) control algorithm. The equations of motion form a nonlinear ODE-PDE system, with ODE describing bus rotation and PDE describing solar array flexible dynamics. The attitude control objective was achieved using the inertial coupling between the cylinder and the flexible appendage. The control law computes the beam dynamics required to achieve the desired attitude and actuates the flexible beam using the distributed actuation using an actuator to control mapping. The attitude tracking control law is designed in terms of a variable $\delta$ that scales with the stiffness term to simulate an uncanceled or partially canceled beam dynamics. The exponential stability of the closed-loop system for $\delta=1$ corresponding to full beam dynamics cancellation with a feed-forward term was proven. The closed-loop system was simulated for different $\delta$ values to verify 
trajectory tracking and slewing. To test the control algorithms in real-time, we developed an experimental setup. The setup includes a cylinder and a beam connected using an interference fit. Strain actuation in the beam was achieved using piezoelectric actuators (PZT). The quasi-static actuator model used for SASA experiments was validated by comparing tip deflections of a cantilever beam obtained from simulations against experimental measurements. We achieved controlled cylinder rotation using beam strain-actuation on the experimental setup, which validates the SASA control algorithm. Strain-actuation was extended to a distributed actuation configuration with multiple PZTs. This model was used to test the controllers in a distributed configuration for trajectory tracking and slewing.

\section{Acknowledgments}

Government sponsorship is acknowledged. The research was in part carried out at the Jet Propulsion Laboratory, California Institute of Technology under a contract with the National Aeronautics and Space Administration. The authors acknowledge help from Kevin Lohan in the design and implementation of the experimental setup.

\section{References}

1 Andersen, G., Quinn, D., Beals, G., Nelson, J., and Nurre, G., "An Overview of the Hubble Space Telescope Pointing Control System Design and Operation," Astrodynamics Conference, American Institute of Aeronautics and Astronautics, aug 1992, doi: 10.2514/6.1992-4616

2 Meza, L., Tung, F., Anandakrishnan, S., Spector, V., and Hyde, T., "Line Of Sight Stabilization for the James Webb Space Telescope," AAS Guidance, and Control Conference, Breckenridge, Colorado, Feb. 2005 .

3 Knapp, M., Smith, M., Pong, C., Nash, J., and Seager, S., "ExoplanetSat: High Precision Photometry for Exoplanet Transit Detections in a 3U," IAC 16th Symposium on Small Satellite Missions, 2012, IAC-12-B4.

4 Stapelfeldt, K., Belikov, R., Bryden, G., Cahoy, K., Chakrabarti, S., Marley, M., McElwain, M., Meadows, V., Serabyn, E., Trauger, J., et al., "Exo-C Imaging Nearby Worlds," by the Science and Technology Definition Team (STDT) and the Exo-C Design team, final report, https: //exoplanets.nasa.gov/stdt/ExoC_InterimReport.pdf, 2015.

5 Guelman, M., Kogan, A., Kazarian, A., Livne, A., Orenstein, M., and Michalik, H., "Acquisition and 
Pointing Control for Inter-Satellite Laser Communications," IEEE Transactions on Aerospace and Electronic Systems, Vol. 40, No. 4, Oct 2004, pp. 1239-1248, doi: 10.1109/TAES.2004.1386877

${ }^{6}$ Bandyopadhyay, S., Foust, R., Subramanian, G. P., Chung, S.-J., and Hadaegh, F. Y., "Review of Formation Flying and Constellation Missions Using Nanosatellites," Journal of Spacecraft and Rockets, Vol. 53, No. 3, may 2016, pp. 567-578, doi: 10.2514/1.a33291

7 Chung, S.-J., "ARGOS Testbed: Study of Multidisciplinary Challenges of Future Spaceborne Interferometric Arrays," Optical Engineering, Vol. 43, No. 9, sep 2004, pp. 2156, doi: 10.1117/1.1779232

8 Blackmore, L., Murray, E., Scharf, D. P., Aung, M., Bayard, D., Brugarolas, P., Hadaegh, F., Lee, A., Milman, M., Sirlin, S., et al., "Instrument Pointing Capabilities: Past, Present, and Future," AAS Guidance and Control Conference, Breckenridge, Colorado, Feb. 2011.

9 Chilan, C. M., Herber, D. R., Nakka, Y. K., Chung, S.-J., Allison, J. T., Aldrich, J. B., and AlvarezSalazar, O. S., "Co-Design of Strain-Actuated Solar Arrays for Spacecraft Precision Pointing and Jitter Reduction," AIAA Journal, Vol. 55, No. 9, sep 2017, pp. 3180-3195, doi: 10.2514/1.j055748

10 Alvarez-Salazar, O., Aldrich, J., Filipe, N., Allison, J., and Chung, S.-J., "Strain Actuated Solar-Arrays for Precision Pointing of Spacecraft," AAS Guidance, Navigation, and Control Conference, Breckenridge, Colorado, Feb. 2016.

11 Chilan, C. M., Herber, D. R., Nakka, Y. K., Chung, S.-J., Allison, J. T., Aldrich, J. B., and AlvarezSalazar, O. S., "Co-Design of Strain-Actuated Solar Arrays for Precision Pointing and Jitter Reduction," 5\%th AIAA/ASCE/AHS/ASC Structures, Structural Dynamics, and Materials Conference, American Institute of Aeronautics and Astronautics, jan 2016, doi: 10.2514/6.2016-0162

12 Meirovitch, L., Dynamics and Control of Structures, John Wiley \& Sons, 1990.

13 Hodges, D. H. and Pierce, G. A., "Introduction to Structural Dynamics and Aeroelasticity," Introduction to Structural Dynamics and Aeroelasticity, Cambridge University Press, doi: $10.1017 /$ cbo9780511997112.002

14 Meirovitch, L. and Stemple, T., "Hybrid Equations of Motion for Flexible Multibody Systems Using Quasicoordinates," Journal of Guidance, Control, and Dynamics, Vol. 18, No. 4, jul 1995, pp. 678-688, doi: $10.2514 / 3.21447$

15 Junkins, J. L. and Kim, Y., Introduction to Dynamics and Control of Flexible Structures, American Institute of Aeronautics and Astronautics, jan 1993, doi: 10.2514/4.862076

36 of 39 
16 Piedboeuf, J., "Six Methods to Model a Flexible Beam Rotating in the Vertical Plane," Proceedings 2001 ICRA. IEEE International Conference on Robotics and Automation (Cat. No.01CH37164), IEEE, doi: 10.1109/robot.2001.933051

17 Kane, T. R., Ryan, R., and Banerjee, A. K., "Dynamics of a Cantilever Beam Attached to a Moving Base," Journal of Guidance, Control, and Dynamics, Vol. 10, No. 2, mar 1987, pp. 139-151, doi: 10.2514/3.20195

18 Modi, V., Ng, A., and Karray, F., "Nonlinear Dynamics and Control of Orbiting Structures: An Approach with Applications," Proceedings of IEEE International Conference on Control and Applications, IEEE, doi: $10.1109 /$ cca. 1993.348315

19 Spong, M. W., Underactuated Mechanical Systems, Springer Berlin Heidelberg, Berlin, Heidelberg, 1998, pp. $135-150$.

20 Chung, S.-J., Slotine, J.-J. E., and Miller, D. W., "Propellant-Free Control of Tethered Formation Flight, Part 2: Nonlinear Underactuated Control," Journal of Guidance, Control, and Dynamics, Vol. 31, No. 5, sep 2008, pp. 1437-1446, doi: 10.2514/1.32189

21 Herber, D. R., McDonald, J. W., Alvarez-Salazar, O. S., Krishnan, G., and Allison, J. T., "Reducing Spacecraft Jitter During Satellite Reorientation Maneuvers via Solar Array Dynamics," AIAA/ISSMO Multidisciplinary Analysis and Optimization Conference, No. AIAA 2014-3278, Atlanta, GA, USA, June 2014, doi: 10.2514/6.2014-3278

22 Balas, M. J., "Active Control of Flexible Systems," Journal of Optimization Theory and Applications, Vol. 25, No. 3, July 1978, pp. 415-436, doi: 10.1007/BF00932903

23 Meirovitch, L. and Baruh, H., "On the Problem of Observation Spillover in Self-Adjoint DistributedParameter Systems," Journal of Optimization Theory and Applications, Vol. 39, No. 2, Feb 1983, pp. 269291, doi: $10.1007 / \mathrm{BF} 00934533$

$24 \mathrm{He}, \mathrm{W}$. and Ge, S. S., "Dynamic Modeling and Vibration Control of a Flexible Satellite," IEEE Transactions on Aerospace and Electronic Systems, Vol. 51, No. 2, April 2015, pp. 1422-1431, doi: $10.1109 /$ TAES.2014.130804

25 Song, G. and Agrawal, B. N., "Vibration Suppression of Flexible Spacecraft During Attitude Control," Acta Astronautica, Vol. 49, No. 2, jul 2001, pp. 73-83, doi: 10.1016/s0094-5765(00)00163-6

26 Christofides, P. D. and Daoutidis, P., "Finite-Dimensional Control of Parabolic PDE Systems Using Approximate Inertial Manifolds," Proceedings of the 36th IEEE Conference on Decision and Control, Vol. 2, Dec 1997, pp. 1068-1073 vol.2, doi: 10.1109/CDC.1997.657588

37 of 39

American Institute of Aeronautics and Astronautics 
27 Curtain, R. F. and Zwart, H., An Introduction to Infinite-Dimensional Linear Systems Theory, Springer New York, 1995, doi: 10.1007/978-1-4612-4224-6

28 Krstic, M. and Smyshlyaev, A., Boundary Control of PDEs, SIAM, Jan 2008, doi: $10.1137 / 1.9780898718607$

29 Artstein, Z., "Linear Systems with Delayed Controls: A Reduction," IEEE Transactions on Automatic Control, Vol. 27, No. 4, Aug 1982, pp. 869-879, doi: 10.1109/TAC.1982.1103023

30 Kwon, W. and Pearson, A., "Feedback Stabilization of Linear Systems with Delayed Control," IEEE Transactions on Automatic Control, Vol. 25, No. 2, Apr 1980, pp. 266-269, doi: $10.1109 /$ TAC.1980.1102288

31 Krstic, M., "Compensating a String PDE in the Actuation or Sensing Path of An Unstable ODE," 2009 American Control Conference, June 2009, pp. 4097-4102, doi: 10.1109/ACC.2009.5159876

32 Tang, S. and Xie, C., "Stabilization of a Coupled PDE-ODE System by Boundary Control," 49th IEEE Conference on Decision and Control, Dec 2010, pp. 4042-4047, doi: 10.1109/CDC.2010.5718141

33 Krstic, M., "Compensating Actuator and Sensor Dynamics Governed by Diffusion PDEs," Systems \& Control Letters, Vol. 58, No. 5, may 2009, pp. 372-377, doi: 10.1016/j.sysconle.2009.01.006

34 Susto, G. A. and Krstic, M., "Control of PDE-ODE Cascades with Neumann Interconnections," Journal of the Franklin Institute, Vol. 347, No. 1, feb 2010, pp. 284-314, doi: 10.1016/j.jfranklin.2009.09.005

35 Spong, M. W., Hutchinson, S., and Vidyasagar, M., Robot Modeling and Control, Vol. 3, Wiley New York, 2006.

36 Fati-Saber, R., "Nonlinear Control and Reduction of Underactuated Systems with Symmetry. I. Actuated Shape Variables Case," Proceedings of the 40th IEEE Conference on Decision and Control (Cat. No.01CH37228), IEEE, doi: 10.1109/cdc.2001.980834

37 Slotine, J.-J. E. and Li, W., "On the Adaptive Control of Robot Manipulators," Int. J. Rob. Res., Vol. 6, No. 3, Sept. 1987, pp. 49-59, doi: $10.1177 / 027836498700600303$

38 Slotine, J.-J. E. and Li, W., Applied Nonlinear Control, Pearson, Upper Saddle River, NJ, 1991.

39 Bisplinghoff, R. L., Ashley, H., and Halfman, R. L., Aeroelasticity, Addison-Wesley, 1995.

40 Paranjape, A. A., Chung, S.-J., Hilton, H. H., and Chakravarthy, A., "Dynamics and Performance of Tailless Micro Aerial Vehicle with Flexible Articulated Wings," AIAA Journal, Vol. 50, No. 5, May 2012, pp. 1177-1188, doi: 10.2514/1.J051447

38 of 39

American Institute of Aeronautics and Astronautics 
41 Paranjape, A. A., Guan, J., Chung, S.-J., and Krstic, M., "PDE Boundary Control for Flexible Articulated Wings on a Robotic Aircraft," IEEE Transactions on Robotics, Vol. 29, No. 3, June 2013, pp. 625-640, doi: $10.1109 /$ TRO.2013.2240711

42 Rao, S. S. and Sunar, M., "Piezoelectricity and Its Use in Disturbance Sensing and Control of Flexible Structures: A Survey," Applied Mechanics Reviews, Vol. 47, No. 4, April 1994, pp. 113-123, doi: $10.1115 / 1.3111074$

43 Bailey, T. and Hubbard Jr., J. E., "Distributed Piezoelectric-Polymer Active Vibration Control of a Cantilever Beam," Journal of Guidance, Control, and Dynamics, Vol. 8, No. 5, Sept. 1985, pp. 605-611, doi: $10.2514 / 3.20029$

44 Crawley, E. F. and Luis, J. D., "Use of Piezoelectric Actuators as Elements of Intelligent Structures," AIAA Journal, Vol. 25, No. 10, Oct. 1987, pp. 1373-1385, doi: 10.2514/3.9792

45 Fanson, J. L. and Chen, J. C., "Structural Control by the Use of Piezoelectric Active Members," NASALangley Research Center NASA/DOD Control/Structures Interaction Technology, 1986, pp. 809-829.

46 Moheimani, S. O. M. and Fleming, A. J., Piezoelectric Transducers for Vibration Control and Damping, Springer, 1st ed., 2006, doi: 10.1007/1-84628-332-9

47 Alvarez-Salazar, O. S. and Iliff, K., "Destabilizing Effects of Rate Feedback on Strain Actuated Beams," Journal of Sound and Vibration, Vol. 221, No. 2, March 1999, pp. 289-307, doi: 10.1006/jsvi.1998.2010

48 Crawley, E. F. and Anderson, E. H., "Detailed Models of Piezoceramic Actuation of Beams," Journal of Intelligent Material Systems and Structures, Vol. 1, No. 1, Jan 1990, pp. 4-25, doi: $10.1177 / 1045389 \times 9000100102$

49 Nakka, Y. K., Nonlinear Attitude Control of Spacecraft With Strain-Actuated Solar Arrays, M.S. Thesis, University of Illinois at Urbana-Champaign, Urbana, IL, USA, Aug. 2016.

50 Sharf, I., "Geometric Stiffening in Multibody Dynamics Formulations," Journal of Guidance, Control, and Dynamics, Vol. 18, No. 4, Jul 1995, pp. 882-890, doi: $10.2514 / 3.21473$

51 Khalil, H. K., Nonlinear systems, 3rd, New Jewsey, Prentice Hall, 2002. 